\title{
Multiple target drug cocktail design for attacking the core network markers of four cancers using ligand-based and structure-based virtual screening methods
}

Yung-Hao Wong ${ }^{1,3}$, Chih-Lung Lin², Ting-Shou Chen², Chien-An Chen², Pei-Shin Jiang ${ }^{2}$, Yi-Hua Lai ${ }^{3}$, Lichieh Julie Chu ${ }^{4}$, Cheng-Wei Li ${ }^{1}$, Jeremy JW Chen ${ }^{3 *}$, Bor-Sen Chen ${ }^{1 *}$

From Joint 26th Genome Informatics Workshop and Asia Pacific Bioinformatics Network (APBioNet) 14th International Conference on Bioinformatics (GIW/InCoB2015)

Tokyo, Japan. 9-11 September 2015

\begin{abstract}
Background: Computer-aided drug design has a long history of being applied to discover new molecules to treat various cancers, but it has always been focused on single targets. The development of systems biology has let scientists reveal more hidden mechanisms of cancers, but attempts to apply systems biology to cancer therapies remain at preliminary stages. Our lab has successfully developed various systems biology models for several cancers. Based on these achievements, we present the first attempt to combine multiple-target therapy with systems biology.

Methods: In our previous study, we identified 28 significant proteins-i.e., common core network markers-of four types of cancers as house-keeping proteins of these cancers. In this study, we ranked these proteins by summing their carcinogenesis relevance values (CRVs) across the four cancers, and then performed docking and pharmacophore modeling to do virtual screening on the $\mathrm{NCl}$ database for anti-cancer drugs. We also performed pathway analysis on these proteins using Panther and MetaCore to reveal more mechanisms of these cancer house-keeping proteins.
\end{abstract}

Results: We designed several approaches to discover targets for multiple-target cocktail therapies. In the first one, we identified the top 20 drugs for each of the 28 cancer house-keeping proteins, and analyzed the docking pose to further understand the interaction mechanisms of these drugs. After screening for duplicates, we found that 13 of these drugs could target 11 proteins simultaneously. In the second approach, we chose the top 5 proteins with the highest summed CRVs and used them as the drug targets. We built a pharmacophore and applied it to do virtual screening against the Life-Chemical library for anti-cancer drugs. Based on these results, wet-lab bioscientists could freely investigate combinations of these drugs for multiple-target therapy for cancers, in contrast to the traditional single target therapy.

Conclusions: Combination of systems biology with computer-aided drug design could help us develop novel drug cocktails with multiple targets. We believe this will enhance the efficiency of therapeutic practice and lead to new directions for cancer therapy.

\footnotetext{
* Correspondence: jwchen@dragon.nchu.edu.tw; bschen@ee.nthu.edu.tw 'Laboratory of Control and Systems Biology, Department of Electrical Engineering, National Tsing Hua University, Hsinchu 30013, Taiwan ${ }^{3}$ Institute of Biomedical Science, National Chung Hsing University, Taiwan 40227, Republic of China

Full list of author information is available at the end of the article
}

(C) 2015 Wong et al. This is an Open Access article distributed under the terms of the Creative Commons Attribution License (http:// creativecommons.org/licenses/by/4.0), which permits unrestricted use, distribution, and reproduction in any medium, provided the original work is properly cited. The Creative Commons Public Domain Dedication waiver (http://creativecommons.org/publicdomain/ zero/1.0/) applies to the data made available in this article, unless otherwise stated. 


\section{Background}

Cancer is the leading cause of human death worldwide. It is a complex set of diseases, and people have tried to reveal its underlying mechanisms to guide the development of novel therapy strategies. In the last two decades, cancer researchers have generated an abundance of knowledge about cancer, and revealed the etiology of various cancers at DNA, RNA, and protein levels [1]. Weinberg et al. summarized the first and next generation of cancer hallmarks to expand the current understanding of the basic mechanisms of cancer $[2,3]$. Recently, due to the scale up in high throughput data, availability of integrated OMICS data, and various advanced statistical analysis methods, many novel systems biology approaches have been employed to reveal the deeper underlying systematic mechanisms of various cancers [4-6].

Traditional computer-aided drug design (CADD) focuses on a single target for therapy, such as Src, FAK, and EGFR in the case of cancer $[7,8]$. Researchers have used virtual screening with de novo methods to develop small molecules that in most cases inhibit these targets (although sometimes they are agonists), and accordingly reduce the expression of these proteins to kill cancer cells. CADD has a long history and many successful examples. CADD methods can be divided into structurebased and ligand-based methods [9]. Methods in the former category analyze both the structures of the target protein and the small molecule inhibitors to design drugs: examples include the docking method and molecular dynamics simulations. On the other hand, methods in the latter category use only the structures of the small molecule inhibitors (drugs) to do statistical calculations to determine the relationship between a drug's $\mathrm{IC}_{50}$ and its corresponding molecular properties: examples include HypoGen pharmacophore modeling, COMFA (and COMSIA) [10], and many other machine learning and regression methods $[11,12]$.

One of the main differences between Western and Chinese medical philosophy is that the former focuses on single targets, while the latter focuses on multiple targets simultaneously [13]. Systems biology reconstructs the regulatory relationships within genetic, metabolic, and protein-protein interaction networks. These biological networks are highly complex, so robustness and sensitivity are their key system-level features $[14,15]$. The intertwined nature of these networks shows us that inhibiting a single protein directly is not the only way to depress its expression. Systems biology will helps us to identify several protein targets to be inhibited simultaneously: due to their network behaviour, this multi-target approach will produce the same or better effect, than focusing on a single target protein. Also, recent research has demonstrated that to inhibit protein-protein interactions (PPI) is another novel anti-cancer strategy [16]. Inspired by the above ideas, based on the result of our previous systems biology studies [17], we have developed a novel multitarget cocktail therapy to focus on common core network markers of four different cancers. Our strategy is different from Dr. Ho's famous cocktail therapy for AIDS [18], which is not targeted therapy. Our method is to apply traditional CADD methods simultaneously to multiple drug targets. We regard this as a great advance in novel anti-cancer strategies.

Theoretical biological background: Recently, PPI-based analysis seems to have become a novel strategy or cancer target drug therapy and the development of precision medicine $[16,19]$. Unlike traditional target drug design, which focuses on the inhibition or activation of a single target protein, usually a receptor or enzyme, PPI-based drug design involves inhibition of PPIs interface that mediate many important biological processes by small molecule; it is a novel and creative approach to drug discovery, especially for anti-cancer. Many clinical and elementary biological researches have concluded that the identification of PPI nodes and hubs that are significant for cell transformation functions in cancer. These PPIs related to cancers have become important targets for cancer therapy. Progress on technologies in the identification of PPI modulators and the clinical validation of the PPI pairs has made anti-cancer therapeutics by interfering with PPIs a reality $[16,19]$.

So, to identify PPI interface and PPI targets are regarded as the future topics for next generation anticancer strategies. Nevertheless, the cancer PPI networks are always highly complex and differ between cancer subtypes. Hence, we put concentration on common PPI network markers and their PPIs with a critical carcinogenesis relevance value (CRV), which is an estimate of the PPI evolution during the carcinogenesis process, to focus on the conservation of house-keeping proteins and their PPI interface characteristics as important targets shared by different cancers. This allows us to not only find out the crucial common pathways of different cancers in carcinogenesis, but also discover novel PPI targets for cancer therapy. Specific network markers can be regarded to represent specific PPI targets for each cancer. To target PPIs in both common core network markers and specific network markers simultaneously may provide new directions for anticancer therapy strategies.

Theoretical, mathematical, and statistical method: The traditional methods to find hubs or driven proteins in the gene regulatory or PPI networks of cancers is different from our method. In our previous study [17], we compared between the networks of each cancer and its corresponding normal PPI, which were obtained by the AIC (akaike information criterion) order detection and 
Student t-test methods from microarray expression data of patients and normal people, respectively, to get the PPI differential network in order to reveal PPI alternations during the tumorigenesis process. And then, we developed a carcinogenesis relevance value (CRV) for each protein in the PPI differential network based on the total alternations of PPI interaction abilities with other proteins to approve the critical PPI changes during tumorigenesis process. In the end, we obtained the core and specific network markers by using the intersection and difference of these 4 cancers with top CRVs. These novel core and specific network markers could provide possible PPI targets for small-molecule drugs to interfere and then destroy tumors. Calculations and estimations were using real microarray expression data. The maximum likelihood parameter estimation method and AIC model order selection method are well-known and widely used system identification methods from experimental data. During these estimation and learning processes, the PPI interaction mathematical model can derive the most probable PPI network for cancer and normal patients from large amount of microarray data and big databases to interpret the hidden biological mechanisms. The above paragraphs are adapted from our previous study [17] to make this paper be a complete paper.

In our previous study, we analyzed various cancersspecifically, bladder, colorectal, liver, and lung cancersthrough regression modeling, microarray data, maximum likelihood parameter estimations, and big database mining. Based on known PPI information and gene expression data from normal and patient samples, we built a cancer PPI network [17]. Here, we focus on not only the PPI networks of single cancer types but also on common core network markers of four different cancers through the intersection of their respective PPI networks. Cancer is a complex disease, and so we tried to reveal house-keeping proteins significant in different cancers, i.e. the common core network markers shared by the different cancers. As the first trial to develop multiple-target therapy, cancer house-keeping proteins (common core network markers) may be a good choice. There are two main parts of this research. One is to find the core network markers of four cancers by systems biology approach, and the other is to attach these network markers by ligand-based and structure-based CADD (computer-aided drug design) methods. The first part has been published on Journal of Theoretical Biology [17]. The whole work of this research is the Part-II, i.e., using the CADD methods to attach the network biomarkers. To make this paper complete, so we described how to get these network biomarkers in our previous research [17].

\section{Materials and methods}

Identification of the core network markers of four cancers (28 proteins) - A brief review of our previous methods

In our previous study [17], we used the systems biology approach to study network markers of various cancers. Firstly, microarray data, PPI databases and PPI interaction models were employed to construct the PPI networks of normal and cancer cells by the maximum likelihood parameter estimation method (see Additional file 1). The AIC system order detection method (Additional file 2) was employed to prune false-positive PPIs to obtain real PPI networks of normal and cancer cells: in other words, we used the reverse engineering method to construct the PPI networks of normal and cancer cells. Then, the differential PPI network-obtained by contrasting the cancer PPI network and normal PPI networkwas used to investigate PPI variations of each protein in the differential PPI network due to carcinogenesis. Finally, the carcinogenesis value (CRV) based on PPI variations was proposed to evaluate the significance of each protein for carcinogenesis in the differential PPI network. Proteins with a significant CRV ( $p$-value $<0.01$ ) were considered to be significant for the progress of the cancer. The complete mathematical model is described as follows.

After organizing the cancer microarray data and PPI data, we used a PPI model, the maximum likelihood parameter estimation method and a model order detection method together to prune each candidate PPIN by the corresponding microarray data to approach the actual PPIN of each cancer. Here, the PPIs of a target protein $i$ in the candidate PPIN can be depicted by the following protein association model:

$$
x_{i}[n]=\sum_{j=1}^{M_{i}} \alpha_{i j} x_{j}[n]+\omega_{i}[n]
$$

where $x_{i}[n]$ represents the expression level of the target protein $i$ for the sample $n ; x_{j}[n]$ represents the expression level of the $j$-th protein interacting with the target protein $i$ for the sample $n$; $\alpha_{i j}$ denotes the association ability between the target protein $i$ and its $j$-th interactive protein; $M_{i}$ represents the number of proteins interacting with the target protein $i$; and $\omega_{i}[n]$ represents the stochastic noise due to other factors or model uncertainty. The biological meaning of equation (1) is that the expression level of the target protein $i$ is associated with the expression levels of the proteins interacting with it. Consequently, a protein association (interaction) model for each protein in the protein pool can be built.

After constructing equation (1) for the PPI model of each protein in the candidate PPIN, we used the 
maximum likelihood estimation method [20] to identify the association parameters in (1) by microarray data as follows (see Additional file 1):

$$
x_{i}(n)=\sum_{j=1}^{M_{i}} \hat{\alpha}_{i j} x_{j}(n)+w_{i}(n)
$$

where $\hat{\alpha}_{i j}$ is identified by the maximum likelihood estimation method.

Once the association parameters for all proteins in the candidate PPI network were identified for each protein, the true protein associations were determined by pruning the false positive PPIs. Akaike Information Criterion (AIC) [20] and a Student's t-test [21] were employed to achieve model order selection for the pruning of false positive protein associations in $\hat{\alpha}_{i j}$ (see Additional file 2).

After the AIC order detection and use of the Student's t-test to determine $p$-values of $\hat{\alpha}_{i j}$, the false positive PPIs $\hat{\alpha}_{i j}$ in (2) were pruned away and only significant PPIs were refined as follows:

$$
x_{i}(n)=\sum_{j=1}^{M_{i}{ }^{\prime}} \hat{\alpha}_{i j} x_{j}(n)+w_{i}{ }^{\prime}(n), i=1,2 \ldots . . M
$$

where $M_{i}{ }^{\prime} \leq M_{i}$ denotes the number of true PPIs, with the target protein $i$, i.e., a number of $M_{i}-M_{i}{ }^{\prime}$ (or false positives) are pruned in the PPIs of target protein $i$. One protein by one protein (i.e., $i=1,2, \ldots, M$ for all proteins in refined PPIN in (3)) results in refined PPIN

$$
\begin{array}{r}
X(n)=A X(n)+w(n) \\
\text { where } X(n)=\left[\begin{array}{c}
x_{1}(n) \\
x_{2}(n) \\
\vdots \\
x_{M}(n)
\end{array}\right], A=\left[\begin{array}{ccc}
\hat{\alpha}_{11} & \ldots & \hat{\alpha}_{1 M} \\
\vdots & \ddots & \vdots \\
\hat{\alpha}_{M 1} & \cdots & \hat{\alpha}_{M M}
\end{array}\right], w(n)=\left[\begin{array}{c}
w_{1}^{\prime}(n) \\
w_{2}^{\prime}(n) \\
\vdots \\
w_{M}^{\prime}(n)
\end{array}\right]
\end{array}
$$

where the interaction matrix $A$ denotes the PPIs.

If there is no PPI between protein $i$ and protein $j$ or it is pruned by AIC order detection due to the false positive PPIs in the refined PPIN, then $\hat{\alpha}_{i j}=0$. In general, $\hat{\alpha}_{i j}=\hat{\alpha}_{j i}$, but if this is not the case, the larger one was chosen as $\hat{\alpha}_{i j}=\hat{\alpha}_{j i}$ to avoid the situation $\hat{\alpha}_{i j} \neq \hat{\alpha}_{j i}$. The above PPIN construction method was employed to construct the refined PPINs for cancer and non-cancer cells of bladder, colorectal, liver, and lung cancer, respectively.

The interaction matrices A of refined PPINs in (4) for cancer and non-cancer cells of the four cancers were constructed, respectively, as follows:

$$
A_{C}^{k}=\left[\begin{array}{ccc}
\hat{\alpha}_{11, C}^{k} & \cdots & \hat{\alpha}_{1 M, C}^{k} \\
\vdots & \ddots & \vdots \\
\hat{\alpha}_{M 1, C}^{k} & \cdots & \hat{\alpha}_{M M, C}^{k}
\end{array}\right], A_{N}^{k}=\left[\begin{array}{ccc}
\hat{\alpha}_{11, N}^{k} & \cdots & \hat{\alpha}_{1 M, N}^{k} \\
\vdots & \ddots & \vdots \\
\hat{\alpha}_{M 1, N}^{k} & \cdots & \hat{\alpha}_{M M, N}^{k}
\end{array}\right]
$$

where $\mathrm{k}$ = bladder cancer, colorectal cancer, liver cancer, and lung cancer; $A_{C}^{k}$ and $A_{N}^{k}$ denote the interaction matrices of refined PPIN of the $k$-th cancer and noncancer, respectively; $M$ is the number of proteins in the refined PPIN. Therefore, the protein association model for CPPIN and NPPIN in the $k$-th cancer and noncancer can be represented by the following equations according to (4) and (5):

$$
\begin{aligned}
& x_{C}^{k}(n)=A_{C}^{k} x_{C}(n)+w_{C}^{k}(n) \\
& x_{N}^{k}(n)=A_{N}^{k} x_{N}(n)+w_{N}^{k}(n)
\end{aligned}
$$

where $k$ = bladder cancer, colorectal cancer, liver cancer, and lung cancer;

$$
x_{C}^{k}(n)=\left[\begin{array}{llll}
x_{1 C}^{k} & x_{2 C}^{k} & \cdots & x_{M C}^{k}
\end{array}\right]^{T} \text { and } x_{N}^{k}(n)=\left[\begin{array}{llll}
x_{1 N}^{k} & x_{2 N}^{k} & \cdots & x_{M N}^{k}
\end{array}\right]^{T}
$$

denote the vectors of expression levels; $w_{C}^{K}(n)$ and $w_{N}^{K}(n)$ indicate the noise vectors of PPINs in the $k$-th cancer and non-cancer cells, respectively.

The different matrix $A_{C}^{k}-A_{N}^{k}$ of differential PPI network between CPPIN and NPPIN in the $k$-th cancer is defined as:

$$
D^{k}=\left[\begin{array}{ccc}
d_{11}^{k} & \cdots & d_{1 M}^{k} \\
\vdots & \ddots & \vdots \\
d_{M 1}^{k} & \cdots & d_{M M}^{k}
\end{array}\right]=\left[\begin{array}{cccc}
\hat{\alpha}_{11, C}^{k}-\hat{\alpha}_{11, N}^{k} & \cdots & \hat{\alpha}_{1 M, C}^{k}-\hat{\alpha}_{1 M, N}^{k} \\
\vdots & \ddots & \vdots \\
\hat{\alpha}_{M 1, C}^{k}-\hat{\alpha}_{M 1, N}^{k} & \cdots & \hat{\alpha}_{M M, C}^{k}-\hat{\alpha}_{M M, N}^{k}
\end{array}\right]
$$

where $k$ = bladder cancer, colorectal cancer, liver cancer, and lung cancer; $d_{i j}^{k}$ denotes the PPI variation between the $i$-th protein and the $\mathrm{j}$-th protein of differential PPI network by comparing CPPIN with NPPIN in the $k$-th type of cancer; the matrix $D^{k}$ indicates the difference in network structure between CPPIN and NPPIN in the $k$-th type of cancer. In order to investigate carcinogenesis from the difference matrix $D^{k}$ between CPPIN and NPPIN of the $k$-th cancer in (7), a score named the carcinogenesis relevance value (CRV) was presented to quantify the correlation of PPI variations of each protein in $D^{k}$ with the significance of carcinogenesis as follows [22]:

$$
C R V^{k}=\left[\begin{array}{c}
C R V_{1}^{k} \\
\vdots \\
C R V_{i}^{k} \\
\vdots \\
C R V_{M}^{k}
\end{array}\right]
$$

where $C R V_{i}^{k}=\sum_{j=1}^{M}\left|d_{i j}^{k}\right|$, and $k=$ bladder cancer, colorectal cancer, liver cancer, and lung cancer.

The $C R V_{i}^{k}$ in (8) quantifies the differential extent of PPI variations of the $i$-th protein (the absolute sum of the $i$-th row of $D^{k}$ in (7)) and $C R V^{k}$ can differentiate 
CPPIN from NPPIN in the $k$-th cancer. In other words, the $C R V_{i}^{k}$ in (8) could calculate the total PPI variations of the $i$-th protein by comparing the network structure differences between the cancer and non-cancer networks, which can be used to check which proteins are involved with the $k$-th cancer.

In order to investigate which proteins are more likely involved in the $k$-th cancer, we needed to calculate the corresponding empirical $p$-value to determine the statistical significance of $C R V_{i}^{k}$. To determine the observed $p$-value of each $C R V_{i}^{k}$, we repeatedly permuted the network structure of the candidate PPIN of the $k$-th type of cancer as a random network of the $k$-th cancer. Each protein in the random network of the $k$-th type of cancer will have its own CRV to generate a distribution of $C R V_{i}^{k}$ for $k$ = bladder, colorectal, liver, and lung cancer. Although there was random disarrangement of the network structure, the linkages of each protein were maintained, i.e., the proteins with which a particular protein interacted were permuted without changing the total number of protein interactions. This procedure was repeated 100,000 times and the corresponding $p$-value was calculated as the fraction of random network structure in which $C R V_{i}^{k}$ is at least as large as the CRV of the real network structure. According to the distributions of $C R V_{i}^{k}$ of random networks, the $C R V_{i}^{k}$ in (8) with a $p$-value of less than or equal to 0.01 was regarded as a significant CRV and the corresponding protein was determined to be a significant protein in the carcinogenesis of the $k$-th cancer: a protein with a $p$-value $>0.01$ was removed from the list of significant proteins in carcinogenesis (in other words, if the $p$-value of $C R V_{i}^{k}>$ 0.01 , then the $i$-th protein was removed from $C R V^{k}$ in (8) and the remainders in $C R V^{k}$ with $p$-values of CRVs less than 0.01 were significant proteins of the $k$-th cancer). Based on the $p$-value of the CRVs for all proteins $(i=1,2, \ldots, M)$ and the four types of cancer $(k=$ bladder, colorectal, liver, and lung cancer), we generated four lists of significant proteins (Additional file 3: Table S1) for the cancers according to the CRV and the statistical assessment of each significant protein in $C R V^{k}$ in (8). As shown in Table S1, we found 107 significant proteins in bladder cancer, 110 significant proteins in liver cancer, 60 significant proteins in colorectal cancer, and 86 proteins in lung cancer. These proteins have significant PPI changes between the CPPIN and NPPIN in the carcinogenic process for their corresponding cancer and we suspect that they may play important roles in carcinogenesis, warranting further investigation.

The intersection of these significant proteins in the four cancers and their PPIs is known as the core network markers, while the differences of these significant proteins are the unique significant proteins of each cancer and their PPIs in each of the cancers are known as the specific network markers for each cancer. We found that there were 28 significant proteins that could be classified as a core network marker and 26, 4, 24, and 13 significant proteins that were specific network markers of bladder, colorectal, liver, and lung cancer, respectively. The core network and specific network markers for the cancers are described in our previous paper [17]. This insight into the carcinogenic mechanisms of common core and specific network markers in different cancers will be discussed in detail in the following section.

The 28 significant proteins in Figure 1(a) (see also Table 1) are significant proteins shared by the four cancers, and these proteins and their PPIs form the common core network markers of these four cancers. The significant proteins outside of these 28 are specific network markers, distinct for each cancer. Finally, based on these common core network markers and specific network markers, we investigated the mechanisms behind the carcinogenesis process with the help of databases (for example, GO database [23], DAVID database $[24,25]$, and KEGG pathway database $[26,27])$ to find multiple network targets for cancer therapy. Unlike conventional theoretical methods that generate a single mathematical model for a cancer network for detailed theoretical analysis, ours is a systems biology approach to cancer network markers based on real microarray data through reverse engineering, theoretical statistical methods, and data mining in combination with big databases. These features made our method novel and helped produce the significant findings of our paper. The above paragraphs are adapted from our previous study [17] to make this paper to be a complete paper.

\section{Several scenarios to break down the cancer core network} In this study, we used several different approaches to break down the cancer core network. This is the key feature of this research, which confers it novelty. In contrast to traditional single target therapy, we used multiple-target cocktail therapy to attack common core network markers, i.e., to inhibit several key proteins simultaneously instead of inhibiting only one single target. We assume that precisely targeting the core network would reduce the activity of the cancer. Based on this assumption, we believe that attacking core network markers simultaneously will be more efficient than attacking only one target. The first important problem is how to choose the group of key proteins to target. The first two novel approaches below to break down the cancer core network are based on the systems biology approach employed in our previous study [17]. We developed the 


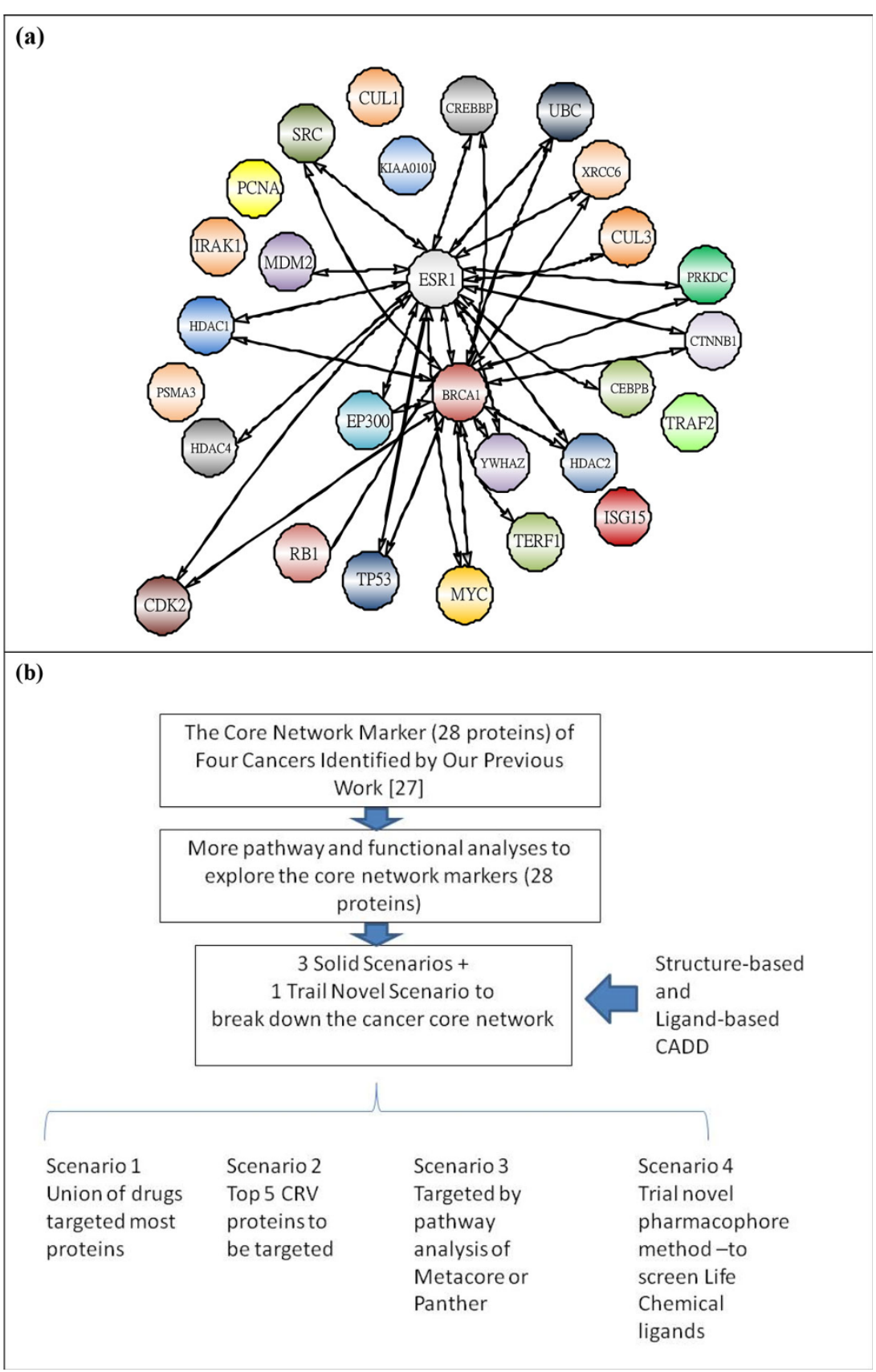

Figure 1 Core network marker and system flow chart. (1a) This is the PPI network of the 28 proteins of the core network marker of four cancers. We see both ESR1 and BRCA1 are most highly connected with other proteins. (1b) We use the CADD method to target the 28 core proteins obtained by previous systems biology method. We use both structure-based and ligand-based CADD with pathway and functional analyses to develop 4 scenarios to break down the core network.

third approach by performing pathway analysis using Panther [28] and MetaCore (GeneGo Inc.) [29]. In addition, we used a 'confusion pharmacophore model' to develop the fourth novel trial approach.

In the first approach, we chose the compounds with highest docking scores for each of the 28 significant proteins. Then we removed the redundant compounds, and analyzed which compounds can target more than two proteins simultaneously. The basic strategy is to minimize the number of compounds necessary to target in order to break down the core network. Since there are 28 proteins in this core network, even choosing only one ligand to inhibit each of these 28 proteins would necessitate 28 ligands, which may be unsuitable for wet-lab validation. 
Table 1. Structure information of $\mathbf{2 8}$ core proteins

\begin{tabular}{|c|c|c|c|c|c|c|c|}
\hline No & Protein & PDB id & Resolution $[\AA ̊]$ & Chains & Length & Lignd & Full Name \\
\hline 1 & BRCA1 & $4 \mid \mathrm{FI}$ & 2.20 & A & 214 & BAAT peptide & Breast cancer type 1 susceptibility protein \\
\hline 2 & CDK2 & 3QQK & 1.86 & A & 306 & $\mathrm{X} 02$ & Cyclin-dependent kinase 2 \\
\hline 3 & CEBPB & * & & & & & CCAAT/enhancer-binding protein beta \\
\hline 4 & CREBBP & 4A9K & 1.81 & $A, B$ & 119 & TYL & CREB-binding protein \\
\hline 5 & CTNNB1 & $3 \mathrm{TX7}$ & 2.76 & A & 527 & P6L & Catenin (cadherin-associated protein), beta 1 \\
\hline 6 & CUL1 & $4 \mathrm{~F} 52$ & 3.00 & $A, C$ & 282 & n.a. & Cullin 1 \\
\hline 7 & CUL3 & $4 \mathrm{EOZ}$ & 2.40 & $B, D$ & 346 & n.a. & Cullin-3 \\
\hline 8 & EP300 & $4 \mathrm{BHW}$ & 2.80 & $A, B$ & 578 & $01 \mathrm{~K}$ & E1A binding protein p300 \\
\hline 9 & ESR1 & $1 \mathrm{UOM}$ & 2.28 & A & 254 & PTI & Estrogen receptor 1 \\
\hline 10 & HDAC1 & $4 B K X$ & 3.00 & B & 482 & n.a. & Histone deacetylase 1 \\
\hline 11 & HDAC2 & $4 \mathrm{LY} 1$ & 1.57 & $A, B, C$ & 369 & $20 Y$ & Histone deacetylase 2 \\
\hline 12 & HDAC4 & 2VQM & 1.80 & A & 413 & HA3 & Histone deacetylase 4 \\
\hline 13 & IRAK1 & * & & & & & Interleukin-1 receptor-associated kinase 1 \\
\hline 14 & ISG15 & 3SDL & 2.29 & C, D & 164 & n.a. & ISG15 ubiquitin-like modifier \\
\hline 15 & KIAA0101 & * & & & & & KIAA0101 \\
\hline 16 & MDM2 & $4 \mathrm{MDN}$ & 1.90 & A & 94 & $\mathrm{Y} 30$ & Mouse double minute 2 homolog \\
\hline 17 & MYC & 1NKP & 1.80 & $A, D$ & 88 & n.a. & V-myc myelocytomatosis viral oncogene homolog \\
\hline 18 & PCNA & $3 W G W$ & 2.80 & $A, B$ & 261 & $\mathrm{~T} 2 \mathrm{~B}$ & Proliferating cell nuclear antigen \\
\hline 19 & PRKDC & $3 K G V$ & 6.60 & $A-F, O, P-T, X-Y$ & 4128 & n.a. & Protein kinase, DNA-activated, catalytic polypeptide \\
\hline 20 & PSMA3 & * & & & & & Proteasome subunit alpha type- 3 \\
\hline 21 & RB1 & ЗРОМ & 2.50 & $A, B$ & 352 & n.a. & Retinoblastoma 1 \\
\hline 22 & SRC & $2 S R C$ & 1.50 & A & 452 & ANP & Proto-oncogene tyrosine-protein kinase Src \\
\hline 23 & TERF1 & $3 \mathrm{BQO}$ & 2.00 & A & 211 & TIN2 peptide & Telomeric repeat-binding factor 1 \\
\hline 24 & TP53 & $1 \mathrm{TSR}$ & 2.20 & $A, B, C$ & 219 & n.a. & Tumor protein p53 \\
\hline 25 & TRAF2 & $1 \mathrm{D} 0 \mathrm{~A}$ & 2.00 & $A-F$ & 168 & OX40L peptide & TNF receptor-associated factor 2 \\
\hline 26 & UBC & 4FJV & 2.05 & $B, D$ & 86 & n.a. & Ubiquitin C \\
\hline 27 & $\mathrm{XRCC6}$ & 1JEQ & 2.70 & A & 609 & n.a. & X-ray repair cross-complementing 6 \\
\hline 28 & YWHAZ & $4 \mathrm{HKC}$ & 2.20 & A & 250 & alpha-4 peptide & 14-3-3 protein zeta/delta \\
\hline
\end{tabular}

*3D structures prepared by homology model, I-Tasser.

In the second approach, we used the summation of the CRVs (Table 2 and Table 3 ) as the criterion to choose the key proteins to inhibit. In our previous study, we showed that CRV quantifies the extent of a protein's association with other proteins, and thus it is optimal to target proteins with the highest summation of CRVs. This strategy depends on the cancer being targeted; in the present study, we focused on the common core network markers of the four cancers, so we chose the highest summation of CRVs across the four cancers.

In the third approach, we employed many new and valuable pathway analyses. Wet-lab cancer researchers can choose the pathway they want to focus on as the therapy target. There are many different combinations dependent on different conditions of cancers, so we do not list all the possibilities. Here, we demonstrate a single example, and others can develop their own scenarios following this example with the help of our docking results. (Additional file 4: Table S2)

The fourth novel approach is to develop confusion and individual pharmacophore models of core network markers (28 proteins) to do virtual screening using the compounds stored in the Life Chemical database.

We highlight the different approaches in this section, because they form the crux of this study. Please see Figure (1b). In the following sections, we describe the related methods necessary for each of the approaches.

\section{More pathway and functional analyses}

\section{A. Panther}

In our previous studies, we performed pathway analysis using the DAVID database. Here, we expand on the previous analysis by performing more pathway and functional analyses in order to develop a more efficient strategy for multiple target therapy. This approach will allow us to accumulate more information on possible therapy strategies. The 28 proteins comprising the cancer core network markers were analyzed for their molecular functions, molecular processes, and subcellular localizations by the PANTHER (Protein ANalysis THrough Evolutionary Relationships) classification system [28]. PANTHER was designed to classify proteins (and their 
Table 2. (a): CRVs of the 28 core proteins of each of the 4 cancers [17]

\begin{tabular}{|c|c|c|c|c|c|c|c|}
\hline $\begin{array}{l}\text { Protein } \\
\text { Name } \\
\text { Nam } \\
\end{array}$ & $\begin{array}{l}\text { CRV of Bladder } \\
\text { Cancer }\end{array}$ & $\begin{array}{l}\text { CRV of } \\
\text { Liver } \\
\text { Cancer }\end{array}$ & $\begin{array}{l}\text { CRV of Colorectal } \\
\text { Cancer }\end{array}$ & $\begin{array}{l}\text { CRV of } \\
\text { Lung } \\
\text { Cancer }\end{array}$ & $\begin{array}{l}\text { CRV SUM of } 4 \\
\text { Cancers }\end{array}$ & $\begin{array}{c}\text { Protein } \\
\text { Name }\end{array}$ & $\begin{array}{c}\text { CRV } \\
\text { SUM } \\
\text { (Sorted) }\end{array}$ \\
\hline BRCA1 & 11.5863 & 6.8236 & 10.8636 & 12.5188 & 41.7923 & UBC & 496.3222 \\
\hline CDK2 & 16.7366 & 14.069 & 14.4739 & 9.942 & 55.2215 & TP53 & 79.4445 \\
\hline CEBPB & 5.2303 & 4.433 & 5.4262 & 7.2717 & 22.3612 & KIAA0101 & 71.9887 \\
\hline CREBBP & 12.0995 & 9.5856 & 11.5906 & 11.1892 & 44.4649 & HDAC1 & 70.5646 \\
\hline CTNNB1 & 7.6796 & 9.5993 & 9.304 & 4.8015 & 31.3844 & CDK2 & 55.2215 \\
\hline CUL1 & 13.2669 & 11.2802 & 5.5283 & 8.5044 & 38.5798 & CUL3 & 53.8054 \\
\hline CUL3 & 13.0117 & 12.9519 & 16.3169 & 11.5249 & 53.8054 & MYC & 53.3347 \\
\hline EP300 & 12.1078 & 13.2218 & 8.3187 & 7.1278 & 40.7761 & PCNA & 52.9045 \\
\hline ESR1 & 5.6189 & 10.3758 & 6.3873 & 8.6696 & 31.0516 & HDAC2 & 47.6069 \\
\hline HDAC1 & 19.2879 & 19.5736 & 11.7823 & 19.9208 & 70.5646 & CREBBP & 44.4649 \\
\hline HDAC2 & 11.0938 & 9.7752 & 18.9463 & 7.7916 & 47.6069 & BRCA1 & 41.7923 \\
\hline HDAC4 & 5.8659 & 5.8397 & 9.7845 & 6.2225 & 27.7126 & EP300 & 40.7761 \\
\hline IRAK1 & 4.1157 & 6.646 & 7.0177 & 5.1777 & 22.9571 & CUL1 & 38.5798 \\
\hline ISG15 & 4.4856 & 6.0239 & 5.8124 & 4.943 & 21.2649 & YWHAZ & 35.9252 \\
\hline KIAA0101 & 15.8188 & 16.7663 & 19.1403 & 20.2633 & 71.9887 & CTNNB1 & 31.3844 \\
\hline MDM2 & 4.5647 & 4.753 & 11.5648 & 6.2816 & 27.1641 & ESR1 & 31.0516 \\
\hline MYC & 13.0423 & 10.7821 & 20.0595 & 9.4508 & 53.3347 & SRC & 28.3907 \\
\hline PCNA & 13.3217 & 15.1438 & 9.6282 & 14.8108 & 52.9045 & TRAF2 & 27.7637 \\
\hline PRKDC & 4.0781 & 5.9369 & 8.5589 & 5.6736 & 24.2475 & HDAC4 & 27.7126 \\
\hline PSMA3 & 5.8022 & 7.7978 & 8.0875 & 5.2831 & 26.9706 & MDM2 & 27.1641 \\
\hline RB1 & 6.8922 & 5.5531 & 5.9763 & 8.3205 & 26.7421 & PSMA3 & 26.9706 \\
\hline SRC & 8.8026 & 4.0767 & 9.1407 & 6.3707 & 28.3907 & RB1 & 26.7421 \\
\hline TERF1 & 5.4642 & 4.9046 & 6.2595 & 8.0708 & 24.6991 & XRCC6 & 25.7358 \\
\hline TP53 & 19.5883 & 18.7422 & 25.9329 & 15.1811 & 79.4445 & TERF1 & 24.6991 \\
\hline TRAF2 & 9.2003 & 4.7703 & 7.365 & 6.4281 & 27.7637 & PRKDC & 24.2475 \\
\hline UBC & 158.5321 & 137.284 & 80.3851 & 120.121 & 496.3222 & IRAK1 & 22.9571 \\
\hline XRCC6 & 5.2871 & 9.5585 & 6.0231 & 4.8671 & 25.7358 & CEBPB & 22.3612 \\
\hline YWHAZ & 8.7995 & 12.6421 & 7.9038 & 6.5798 & 35.9252 & ISG15 & 21.2649 \\
\hline
\end{tabular}

Table 3. (b) Top 5 ligands for the top 5 CRV proteins

\begin{tabular}{|c|c|c|c|c|c|c|c|c|c|}
\hline \multicolumn{2}{|c|}{ UBC(4FJV) } & \multicolumn{2}{|c|}{ TP53(1TSR) } & \multicolumn{2}{|c|}{ KIAA0101 } & \multicolumn{2}{|c|}{ HDAC1 (4BKX) } & \multicolumn{2}{|c|}{ CDK2(3QQK) } \\
\hline $\begin{array}{c}\mathrm{NCl} \\
\text { Drug }\end{array}$ & LibDock Score & $\begin{array}{c}\mathrm{NCl} \\
\text { Drug }\end{array}$ & LibDock Score & $\begin{array}{c}\mathrm{NCl} \\
\text { Drug }\end{array}$ & LibDock Score & $\begin{array}{c}\mathrm{NCl} \\
\text { Drug }\end{array}$ & LibDock Score & $\begin{array}{c}\mathrm{NCl} \\
\text { Drug }\end{array}$ & LibDock Score \\
\hline 719481 & 122.887 & 673172 & 126.112 & 655102 & 165.722 & 627865 & 134.685 & 680359 & 108.336 \\
\hline 633409 & 114.187 & 695409 & 125.821 & 669588 & 164.036 & 625439 & 134.097 & 678636 & 101.875 \\
\hline 672968 & 111.867 & 682236 & 117.725 & 407811 & 158.565 & 647638 & 125.505 & 669299 & 101.27 \\
\hline 734999 & 111.578 & 695405 & 115.63 & 704564 & 148.676 & 2426 & 124.722 & 679065 & 101.006 \\
\hline 688121 & 111.501 & 667504 & 114.326 & 698687 & 147.054 & 707841 & 117.076 & 376791 & 100.92 \\
\hline
\end{tabular}

genes) in order to facilitate high-throughput analysis. It has a friendly user interface, you only need to input the gene list and set up the parameters, you will get the results you want. Please see Additional file 5: Figure S5.1 and the PANTHER website [28]

B. MetaCore
MetaCore includes a manually annotated database of gene interactions and metabolic reactions obtained from the scientific literature, including the most newly updated ones. The enrichment analysis of the biological process was based on the hypergeometric distribution algorithm and relevant pathway maps. The mathematical 
foundation of MetaCore is shown in supplementary materials.

More pathway and functional analyses are fundamental to learning more about the hidden mechanisms of cancer networks. So, to interpret the results for the third approach in a meaningful way, this topic must be explained and described beforehand. The novelty of this paper is in its combination of systems biology with computer-aided drug design. Our research opens many new directions for multiple-target drug design; we describe only a portion of our results in this paper, for illustrative purposes. Our results clearly show that our approaches show great promise for future research to target special pathways through the design of drugs having multiple targets.

Metacore is also user-friendly software. The various manuals and training materials can be download from public website [30,31], and due to the copyright concern, we do not copy too many material here. Please follow the instructions in these manuals, and you can very well analysis. The mathematical foundation of Metacore is shown in Additional file 6. Of course, the more deeply you understand the underlying statistics meaning, you can do better analysis.

\section{Protein and ligand structures}

The following section illustrates the computer-aided drug design (CADD) strategy applied to target the 28 common core network proteins. The first through third approaches need both the 3D structures of proteins and ligands, while the fourth pharmacophore approach only needs the structures of the ligands. The first thing we need is thus the 3D structures of the proteins. At this stage, 24 of them are available in the well-known PDB database, and we can download them directly, while there are four proteins (CEBPB, IRAK1, KIAA0101 and PSMA3) whose 3D structures have not been solved at this stage (Table 1). We used the NCI (46872 ligands) and Life Chemical (31742 ligands) drug libraries with the filter "anti-cancer". The Developmental Therapeutics Program NCI/NIH offers the NCI-60 cell line screening. The users can download the 3D ligands (drugs) structures from the website, and after the virtual screening, you can request them send you these drugs for free. Life Chemical is a commercial company, you have to pay to get these drugs [32]. All drug structures were prepared and minimized by Discovery Studio 3.5 (DS3.5). People seldom do the virtual screening on multiple targets since it is computationally intense. We performed this work on an IBM server with 160 cores and 1 TB of memory.

\section{Homology model and binding site prediction}

Proteins do not always have 3D structures available in the PDB database. Since only amino acid sequence data without corresponding structures were available for four of the proteins targeted in our study (CEBPB, IRAK1,
KIAA0101 and PSMA3) we used homology modeling to predict the structures for these proteins. Among many famous software and webservers, the I-Tasser webserver developed by Zhang et al. [33] is the most well-known homology modeling webserver. We used this server to perform homology modeling of our proteins of interest that lacked available 3D structures. For some wellstudied protein targets, structures with embedded ligands are available, so the exact binding site for the docking experiments is known. However, binding site information was unavailable for most proteins, so we used the COACH webserver [34], also developed by Zhang et al., to predict the binding sites. The detailed binding site information is shown in Additional File 7. I-TASSER and COACH are both user friendly webserver. You only need to input the sequence of protein residue into the I-TASSER, and set up the parameters, it will give you the predicted $3 \mathrm{D}$ protein structures. Users input the $3 \mathrm{D}$ protein structures into the $\mathrm{COACH}$ and set up the parameters, and then it will give you the predicted binding sites (Additional file 5: Figure S5.2, S5.3).

\section{Docking}

After prepared the 3D structures of proteins and ligands, and find out the binding site, we can perform docking for virtual screening. We used DS 3.5 to perform docking simulations, and then ranked the docking results based on LibDock score. We chose the top 20 compounds for further analysis, such as the construction of pharmacophore models. The DS 3.5 is also user friendly software, users can download the manual from website, please see the example for parameter setting of docking. Please see Additional file 5: Figure S5.4

\section{Pharmacophore Model}

A. HypoGen method and virtual screening

There are three stages in the generation of the HypoGen model: constructive phase, subtractive phase and optimization phase. (1) Constructive phase: Active ligands within a given range of the maximum activity are chosen initially. The two most active ligands are used to enumerate the pharmacophores. (2) Subtractive phase: After the constructive phase, a database with a large number of pharmacophore structures is generated. The purpose of the subtractive phase is to identify the inactive pharmacophores and eliminate them from the database. (3) Optimization phase: The simulated annealing method is used to modify the scoring function of each hypothesis to be tested. After optimization, the HypoGen method generates the top 10 hypotheses. This study is our first attempt to combine systems biology with CADD. Pharmacophore modeling is one of the most powerful approaches in CADD. Here, we developed a novel prototype pharmacophore model, which is different from the 
traditional one. As mentioned above, this is a novel idea, and the pharmacophore model is just a prototype: it will require further modification in future studies. In traditional pharmacophore modeling, the $\mathrm{IC}_{50}$ value for each drug under investigation is necessary, but our model does not require them. Instead, we linearly transformed LibDock scores to generate putative $\mathrm{IC}_{50}$ values, and used these values to build the pharmacophore. We combined the top three ligands for each of the 28 proteins to make a ligand pool, and then used this ligand pool to construct a common pharmacophore; this is qualitatively different from building 28 individual pharmacophores. We build the Hypogen model by the DS 3.5 , and it is also user friendly. However, to build a correct Hypogen model is a time consuming work, you need to try the different combination of compounds. For a skill expert with normal computational resource, it often needs at least one month to build the correct model. We show the user interface for detailed parameter setting for your reference. Please refer to Additional file 5 : Figure S5.5.

\section{B. PharmaGist}

PharmaGist is another method to construct ligandbased pharmacophores. Because it does not require $\mathrm{IC}_{50}$ values of ligands [35], it is easier to construct pharmacophores by PharmaGist than by the HypoGen model. For each protein, we constructed one PharmaGist model: these models can be used to do virtual screening in the future. PharmaGist is also a user friendly webserver. Please see Additional file 5: Figure S5.6.

\section{Cocktail multiple-target strategy: A novel model combined with systems biology and CADD}

In summary, we used systems biology to construct the common core network marker of four different cancers, which contains 28 proteins as the house-keeping proteins shared by the different cancers. Then we used the docking and pharmacophore methods to perform virtual screening on these 28 core proteins and get the top compounds for each protein. In contrast to traditional single target methods, we suggest using a combination of the top compounds to perform cell proliferation experiments. We have provided an example for each of our first three approaches. Our research provides a novel direction for target therapy for cancers. Wet-lab biomedical scientists can combine the top ligands for each of the 28 core proteins based on their experimental conditions and the pathways that they want to focus on. As an early stage project, we have taken a conservative approach, only using the NCI anti-cancer drug library. As these drugs have proven anti-cancer activity, our cocktail of multiple drugs can be expected to slightly or moderately enhance the therapeutic effect under the right wet-lab conditions. Collaboration with and input from wet-lab experts would permit the modification and optimization of our model, and the scope could expand to include other drug libraries in the virtual screening.

\section{Biological Experimental Validations}

The detailed protocol for the biological experiment in this Part, please refer to our previous work [36]. And the results please refer to Additional file 8. Recently, our team members who are part of wet-lab experiments have found that: if we used more than one drug to attack more than one target at the same time, it will decrease the $\mathrm{IC}_{50}$ of the drug. Our team has shown that if we used Gefitinib (Iressa) and L4 (one drug from the LOPAC drug library) to inhibit Src and EGFR at the same time, we achieve lower cell viability.

\section{Results and Discussion}

\section{Review of the results of our previous methods}

Our previous study identified 28 core proteins in the common core network marker for four cancers [17]. These are the proteins intersecting between the four cancers' PPI networks with the top CRVs. Figure 1(a) shows the PPI information of each common core network marker. As stated previously, these 28 core proteins could be responsible for the house-keeping mechanisms of these four cancers, so using the minimum possible number of ligands to target the maximum possible number of the 28 proteins could be the most efficient strategy to attack the cancers.

\section{Several approaches to break the cancer core network marker}

a. We chose the top 20 compounds with the highest docking scores for each of the 28 significant proteins. Of a total of 560 ligands (Additional file 4: Table S2), we found there were 13 ligands that target 2 or 3 proteins simultaneously (Table 4(a)) after redundancy analysis. These 13 ligands combined 11 target proteins (Table 5new 3(b)). We used the 13 ligands as the minimum package for breaking the core network by inhibiting these 11 proteins. While this does not target all 28 proteins, the main purpose here was to minimize the number of ligands at this first stage trial. (As we have said, this is the first trial of our primary model.) Of course, according to our docking results of these 28 proteins, there are thousands of possible drug combinations that can be used based on different analysis methods as per a given researcher's scope. It is quite possible to develop combinations to target all the 28 proteins based on our docking results.

b. In our previous research, we observed the CRVs of the 28 core proteins for each of the four cancers (Table 2). In this study, we summed up the $4 \mathrm{CRVs}$ for each of the 28 proteins. After ranking these summations, we listed the top 5 CRV summations (Table 2), and suggested them to 
Table 4. $\mathrm{NCl}$ drugs target more than one protein

\begin{tabular}{|c|c|c|c|}
\hline No. & $\mathrm{NCl}$ Drug & Protein Name & LibDock Score \\
\hline \multirow[t]{3}{*}{1} & 625439 & KIAA0101 & 145.708 \\
\hline & 625439 & HDAC1(4BKX) & 134.097 \\
\hline & 625439 & HDAC4(2VQM) & 149.324 \\
\hline \multirow[t]{2}{*}{2} & 645378 & CEBPB(2E_42) & 158.637 \\
\hline & 645378 & KIAA0101 & 140.103 \\
\hline \multirow[t]{2}{*}{3} & 668448 & BRCA1(4IFI) & 192.069 \\
\hline & 668448 & PSMA3 & 166.516 \\
\hline \multirow[t]{2}{*}{4} & 668577 & MYC(1NKP) & 125.603 \\
\hline & 668577 & PSMA3 & 180.11 \\
\hline \multirow[t]{2}{*}{5} & 682094 & TERF1(3BQO) & 123.287 \\
\hline & 682094 & YWHAZ(4HKC) & 182.534 \\
\hline \multirow[t]{2}{*}{6} & 687363 & MYC(1NKP) & 145.138 \\
\hline & 687363 & YWHAZ(4HKC) & 184.649 \\
\hline \multirow[t]{2}{*}{7} & 695175 & TRAF2(1D0A) & 101.267 \\
\hline & 695175 & PSMA3 & 148.83 \\
\hline \multirow[t]{2}{*}{8} & 695409 & TP53(1TSR) & 125.821 \\
\hline & 695409 & PSMA3 & 164.906 \\
\hline \multirow[t]{2}{*}{9} & 704565 & KIAA0101 & 137.38 \\
\hline & 704565 & HDAC4(2VQM) & 153.564 \\
\hline \multirow[t]{2}{*}{10} & 719660 & MYC(1NKP) & 134.021 \\
\hline & 719660 & YWHAZ(4HKC) & 200.903 \\
\hline \multirow[t]{2}{*}{11} & 724305 & CEBPB(2E_42) & 167.724 \\
\hline & 724305 & HDAC4(2VQM) & 141.195 \\
\hline \multirow[t]{2}{*}{12} & 726771 & BRCA1(4IFI) & 142.956 \\
\hline & 726771 & HDAC4(2VQM) & 148.366 \\
\hline \multirow[t]{2}{*}{13} & 742856 & MYC(1NKP) & 140.093 \\
\hline & 742856 & TP53(1TSR) & 100.787 \\
\hline
\end{tabular}

be the therapeutic targets for breaking cancer core network marker. According to our previous systems biology analysis, we believe that inhibiting proteins with the

Table 5. The proposed 13 drugs totally target the following 11 proteins

\begin{tabular}{cc}
\hline No. & Protein Name \\
\hline 1 & BRCA1(4IFI) \\
\hline 2 & CEBPB(2E42) \\
\hline 3 & ISG15(3SDL) \\
\hline 4 & MDM2(4MDN) \\
\hline 5 & PCNA(3WGW) \\
\hline 6 & PRKDC(3KGV) \\
\hline 7 & PSMA3 \\
\hline 8 & SRC(2SRC) \\
\hline 9 & TERF1(3BQO) \\
\hline 10 & TP53(1TSR) \\
\hline 11 & XRCC6(1JEQ)
\end{tabular}

highest CRVs is a highly efficient way to attack cancers. As the first trial, our combination of proteins with the top 5 CRVs should simply be taken as a proof of concept: one can develop combinations according to one's particular needs based on our CRVs.

c. We have also listed pathway analyses using Panther and MetaCore on the core network proteins. Other scientists can use this information to choose the pathways that they want to target, and then choose the best combination of drugs from our virtual screening results. For instance, from the pathway analysis results using Panther (Table 6), researchers interested in targeting the p53 pathway can choose between EP300, CREBBP, HDAC1, HDA2, TRAF2, CDK2, MDM2, and TP53 as the therapy targets. For the above scenario, researchers can choose the top drugs from Additional file 4: Table S2. MetaCore gives us more information for the purpose of target therapy. The top three modules mapped to our 28 core proteins are listed in Table 7. Taking the first map/ module (DNA damage_ATM/ATR regulation of G1/S checkpoint) as an example, we see CDK2, p53, Ubiquitin, BRCA1, c-Myc, PCNA, and MDM2 are related to this module. As above, we can use the drugs in Additional file 4 : Table S2 to choose the optimal combination of drugs to attack this module.

\section{More pathway and functional analysis}

A. Panther

The results of Panther are shown in Figure 2 and listed in Table 6 . We see the 28 core network markers hit many important cancer-related pathways, such as p53, Wnt signaling, p53 feedback loops 2, apoptosis, etc. As we have described above, this study is a prototype model. Inhibition of the right proteins hitting the key pathways is an important strategic consideration in real clinical situations. Our results offer another reference for doctors to design the best combination of multiple inhibitors. In the future, using clinical data from doctors will help us perform deeper analysis. These preliminary results also could help us exclude pathways unrelated to cancer at the first stage, such as those related to Huntington's disease, Alzheimer's disease, etc.

B. MetaCore

The results of MetaCore analysis are described below. We have described the top three maps/modules (Table 7).

(i) DNA damage_ATM/ATR regulation of $\mathrm{G} 1 / \mathrm{S}$ checkpoint (Figure 3): It is the highest scoring map (i.e., the map with the lowest $\mathrm{p}$ value). ATM/ATR regulates both the checkpoints of the G1/S and S/G2. DNA damage checkpoints pathways arrest or delay the progression of cell cycle in response to the DNA damage. Eukaryotic cell cycle have four phases, G1 (G indicating gap), S (Synthesis), G2 (Gap 2), and M (Mitosis), and one outside, G0 (Gap 0) [37]. When DNA damage 
Table 6. the pathway analysis results of Panther

\begin{tabular}{clll}
\hline Rank & \multicolumn{1}{c}{ Pathway title } & Count & \\
\hline 1 & p53 pathway & 8 & EP300,CREBBP,HDAC1,HDA2,TRAF2,CDK2,MDM2,TP53 \\
2 & Wnt signaling pathway & 7 & EP300,CREBBP,HDAC1,MYC,HDAC2,CTNN1,TP53 \\
3 & p53 pathway feedback loops 2 & 6 & MYC,RB1,CTNNB1,CDK2,MDM2,TP53 \\
4 & Parkinson disease & 4 & YWHAZ, CUL1,PSMA3,SRC \\
4 & Gonadotropin releasing hormone receptor pathway & 4 & EP300,CREBBP,CTNNB1, SRC \\
5 & Huntington disease & 3 & EP300,CREBBP,TP53 \\
6 & Apoptosis signaling pathway & 2 & TRAF2,TP53 \\
6 & Angiogenesis & 2 & CTNNB1, SRC \\
6 & P53 pathway feedback loops 1 & 2 & MDM2, TP53 \\
6 & Cadherin signaling pathway & 2 & CTNNB1, SRC \\
6 & Transcription regulation by bZIP transcription factor & 2 & EP300, CREBBP \\
6 & TGF-beta signaling pathway & 2 & EP300, CREBBP \\
7 & Interleukin signaling pathway & 1 & MYC \\
7 & Alzheimer disease-presenilin pathway & 1 & CTNNB1 \\
7 & Integrin signalling pathway & 1 & SRC \\
7 & Insulin/IGF pathway-protein kinase B signaling cascade & 1 & MDM2 \\
7 & Hypoxia response via HIF activation & 1 & CREBBP \\
7 & Ubiquitin proteasome pathway & 1 & MDM2 \\
7 & p53 pathway by glucose deprivation & 1 & TP53 \\
\hline
\end{tabular}

Please see their statistical distribution in Figure 2.

occurs, the G1/S checkpoints will inhibit the initiation of replication to prevent cells from entering the $\mathrm{S}$ phase. They are related to two pathways of signal transduction, to initiate and maintain the $\mathrm{G} 1 / \mathrm{S}$ arrest, respectively [37]. Jiri Bartek et al. discussed "The DNA damage response in tumorigenesis and the treatment of cancer" [38]. [The above description is directly cited from the Metacore document.]

(ii) Transcription_P53 signaling pathway (Figure 4): It is the second highest scoring map (i.e., the map with the second lowest $\mathrm{p}$-value). The Tumor protein $\mathrm{p} 53$, also known as p53 ortransformation-related protein 53 (TRP53), acts a significant role in shielding the genome integrity. While being activated, p53 will bind to the enhancer/promoter regions of downstream target genes. And then it regulates the transcription of these genes, through which it initiates cellular processes that responsible for lots of its tumor-suppressor related functions [39]. It is not surprising that core network of 4 cancers are related to the p53 signaling pathway. [The above description is directly cited from the Metacore document.]

\section{Table 7. Top three modules/maps given by Metacore}

\begin{tabular}{|c|c|c|}
\hline No & Processes & Map of core proteins \\
\hline 1 & $\begin{array}{l}\text { DNA damage_ATM/ATR regulation of G1/S checkpoint: It is the highest scoring map (i.e., the map } \\
\text { with the lowest } \mathrm{p} \text { value). ATM/ATR regulates both the checkpoints of the G1/S and S/G2. DNA damage } \\
\text { checkpoints pathways arrest or delay the progression of cell cycle in response to the DNA damage. } \\
\text { Eukaryotic cell cycle have four phases, G1 (G indicating gap), S (Synthesis), G2 (Gap 2), and M (Mitosis), } \\
\text { and one outside, G0 (Gap 0). }\end{array}$ & $\begin{array}{l}\text { CDK2, p53, Ubiquitin, BRCA1, c-Myc, } \\
\text { PCNA, MDM2 }\end{array}$ \\
\hline 2 & $\begin{array}{l}\text { Transcription_P53 signaling pathway: It is the second highest scoring map (i.e., the map with the } \\
\text { second lowest p-value). The Tumor protein p53, also known as p53 ortransformation-related protein } 53 \\
\text { (TRP53), plays a significant role in shielding the genome integrity. While being activated, p53 will bind } \\
\text { to the enhancer/promoter regions of downstream target genes. And then it regulates the transcription } \\
\text { of these genes, through which it initiates cellular processes that responsible for lots of its tumor- } \\
\text { suppressor related functions. It is not surprising that core network of } 4 \text { cancers are related to the p53 } \\
\text { signaling pathway. }\end{array}$ & $\begin{array}{l}\text { CDK2, p53, CBP, p300, Rb protein, } \\
\text { MDM2, Beta-catenin }\end{array}$ \\
\hline 3 & $\begin{array}{l}\text { DNA damage_BRCA1 as a transcription regulator: It is the third highest scoring map (i.e., the map } \\
\text { with the third lowest p-value). Activation of breast cancer susceptibility gene } 1 \text { (BRCA1) by DNA } \\
\text { damage occurs via activating the ataxia telangiectasia mutated serine/threonine protein kinase (ATM) or } \\
\text { serine/threonine-protein kinase ATR (ataxia telangiectasia and Rad3 related protein kinase). These } \\
\text { protein kinases can either directly or indirectly phosphorylate BRCA1 (by cell cycle checkpoint kinase } 2 \\
\text { (Chk2)). BRCA1 acts a significant role in the DNA repair process by expediting cellular response upon } \\
\text { DNA repair. There are numerous DNA repair pathways (see map } 427 \text { Role of BRCA1 and BRCA2 in DNA } \\
\text { repair). We know that DNA repair is highly related to various cancers. }\end{array}$ & $\begin{array}{l}\text { p53, ESR1 (nuclear), BRCA1, c-Myc, } \\
\text { PCNA, Rb protein }\end{array}$ \\
\hline
\end{tabular}




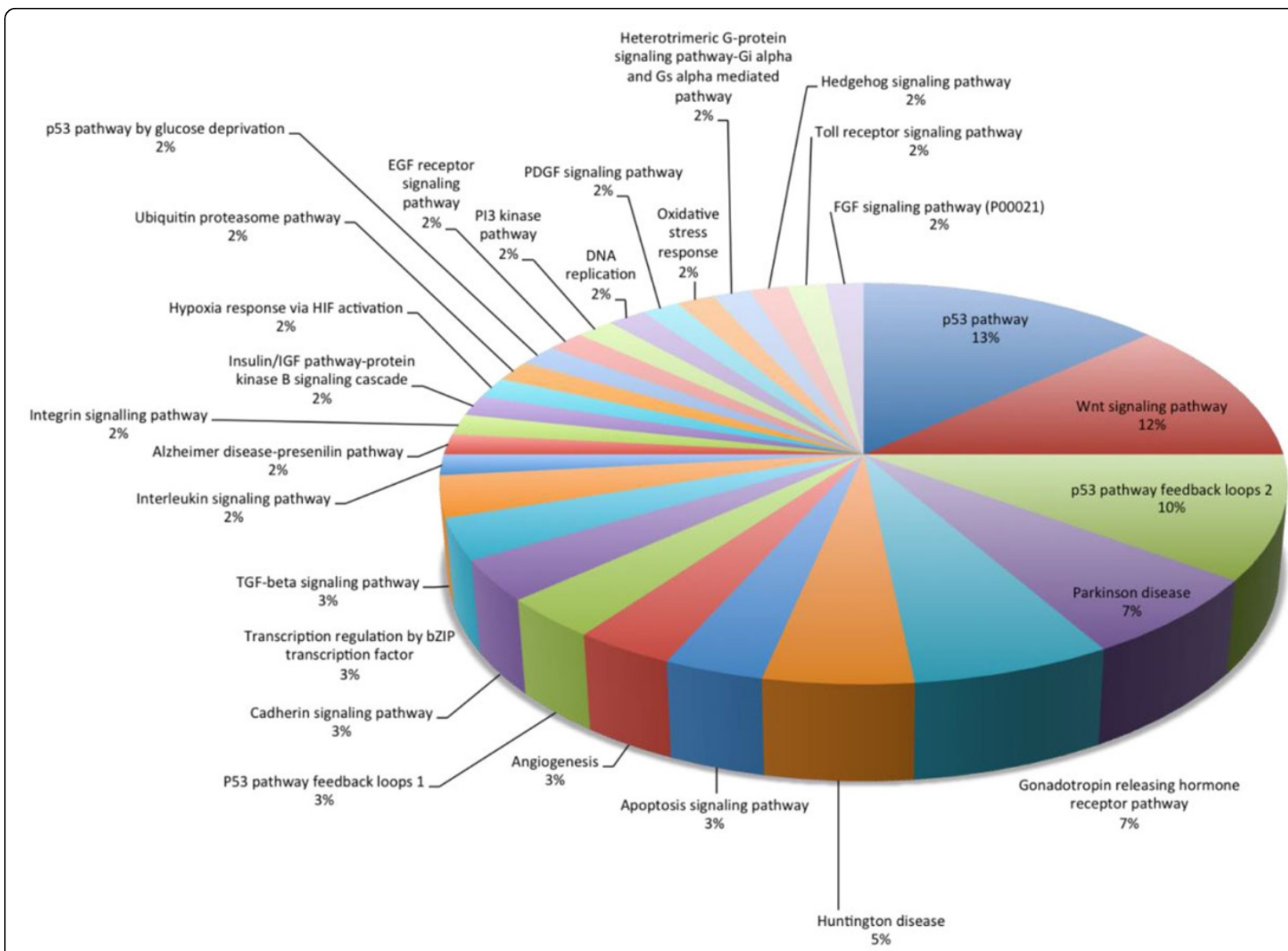

Figure 2 Pathway analysis by Panther. We see the 28 proteins of core network marker can hit many important cancer related pathways, such as p53, Wnt signalling, p53 feedback loops 2, apoptosis, etc. As we have described above, this study is only a prototype model. By the Panther analysis, we could know to inhibit the right proteins which hit the key pathways and is one of the important strategies in the real clinical situation. This result offers another reference for doctors to design the best combination of multiple inhibitors. We will do more deep analysis in the future if more clinical doctors could offer the clinical data to us. The preliminary results also could help us exclude the cancer-unrelated pathways at the first stage, such as Hungtingon disease, alzeheimer, etc.

(iii) DNA damage_Brcal as a transcription regulator (Figure 5): It is the third highest scoring map (i.e., the map with the third lowest $\mathrm{p}$-value). Activation of breast cancer susceptibility gene 1 (BRCA1) by DNA damage occurs via activating the ataxia telangiectasia mutated serine/threonine protein kinase (ATM) [40] or serine/ threonine-protein kinase ATR (ataxia telangiectasia and Rad3 related protein kinase) [41]. These protein kinases can either directly or indirectly phosphorylate BRCA1 (by cell cycle checkpoint kinase 2 (Chk2) [42]). BRCA1 acts a significant role in the DNA repair process by expediting cellular response upon DNA repair. There are numerous DNA repair pathways (see map 427 Role of BRCA1 and BRCA2 in DNA repair) [43]. We know that DNA repair is highly related to various cancers. [The above description is directly cited from the Metacore document.]
* The highest three scoring map (i.e., the map with the three lowest p-value) is based on the enrichment distribution sorted by the 'Statistically significant maps' set. Experimental data from all files is linked to and visualized on the maps as thermometer-like figures. Up-ward thermometers are red and indicate up-regulated signals, and down-ward (blue) ones indicate down-regulated expression levels of the genes. [The above description is directly cited from the Metacore document.]

Statistical analysis results of the three maps are shown in Table 7. These results also help us choose more suitable targets and exclude less suitable ones.

\section{Protein and ligand structures}

Table 1 shows detailed information of these 28 proteins including protein name, PDB ID, resolution, chains, length, ligands, and their full names. Most of these 3D 


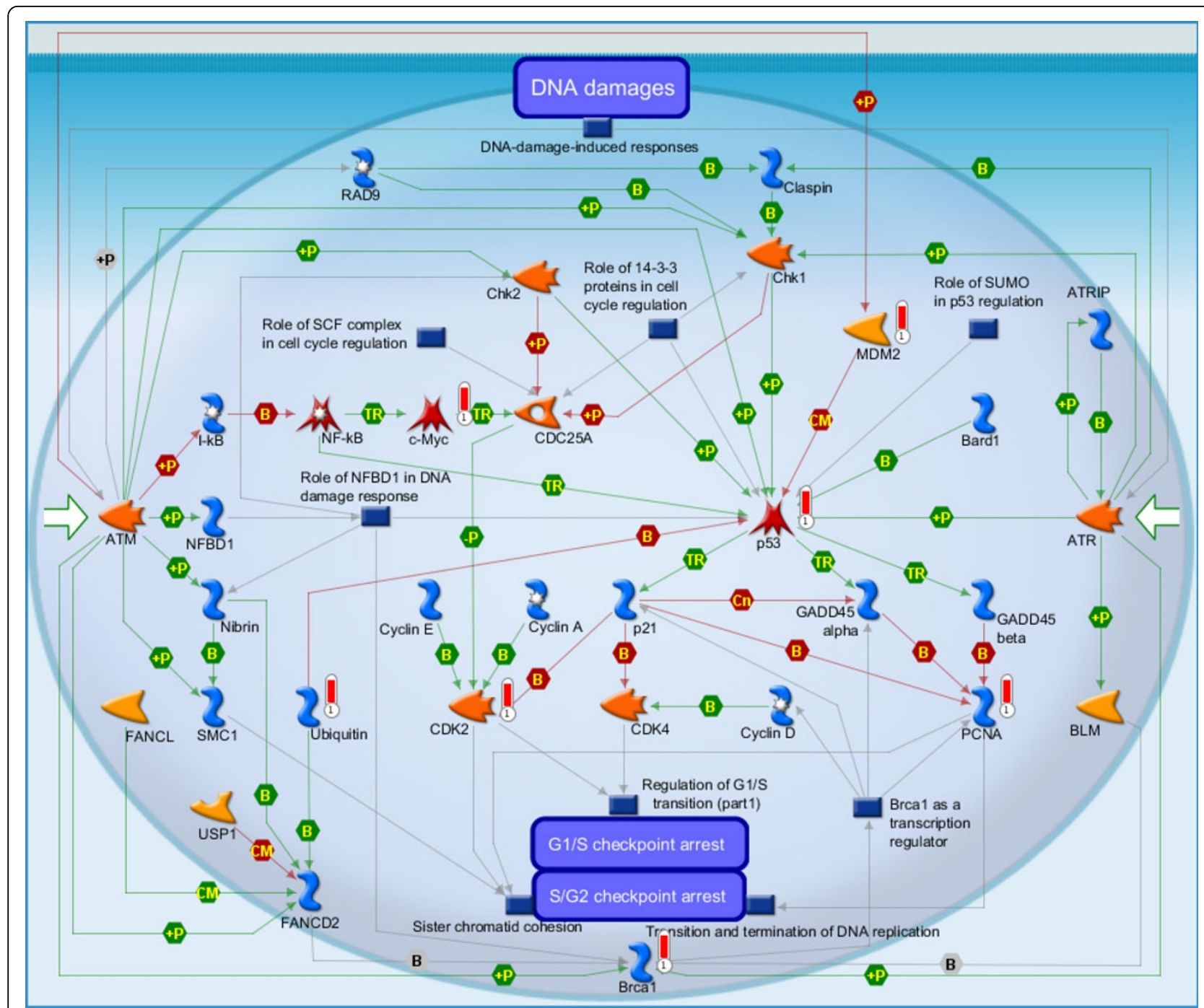

Figure 3 DNA damage_ATM/ATR regulation of G1/S checkpoint. It is the highest scoring map (i.e., the map with the lowest $p$ value). ATM/ ATR regulates both the checkpoints of the G1/S and S/G2. DNA damage checkpoints pathways arrest or delay the progression of cell cycle in response to the DNA damage. Eukaryotic cell cycle have four phases, G1 (G indicating gap), S (Synthesis), G2 (Gap 2), and M (Mitosis), and one outside, G0 (Gap 0).

structures were downloaded from the PDB database. 3D structures of CEBPB, IRAK1, KIAA0101 and PRKDC were constructed by homology modeling using I-TASSER. We can see that many protein structures also contained a ligand in a bound conformation. We used the locations of these ligands within the target protein as the docking site. Binding sites of proteins without bound ligands were predicted using $\mathrm{COACH}$.

\section{Docking}

Figure 6 Additional file 9 shows the docking pose of the top compounds (i.e., those with the highest docking score). Their docking scores ranged from 95 to 241. The analysis also shows the key residues in ligand binding. Our study is the first to perform high-throughput multiple-protein docking, and this work requires large computational resources. Due to space limitations, we have not listed another table for these key residues. The information could be useful for future drug design and these top compounds could be considered as control ligands.

\section{Pharmacophore}

3D pharmacophore modeling is another powerful method to perform virtual screening on large ligand databases. It is as powerful as the docking method, and is always more efficient than docking methods. For example, the large ligands database ZINC [44] has more than 200 million ligands. By calibrating the parameters of the pharmacophore virtual screening, it is possible to 


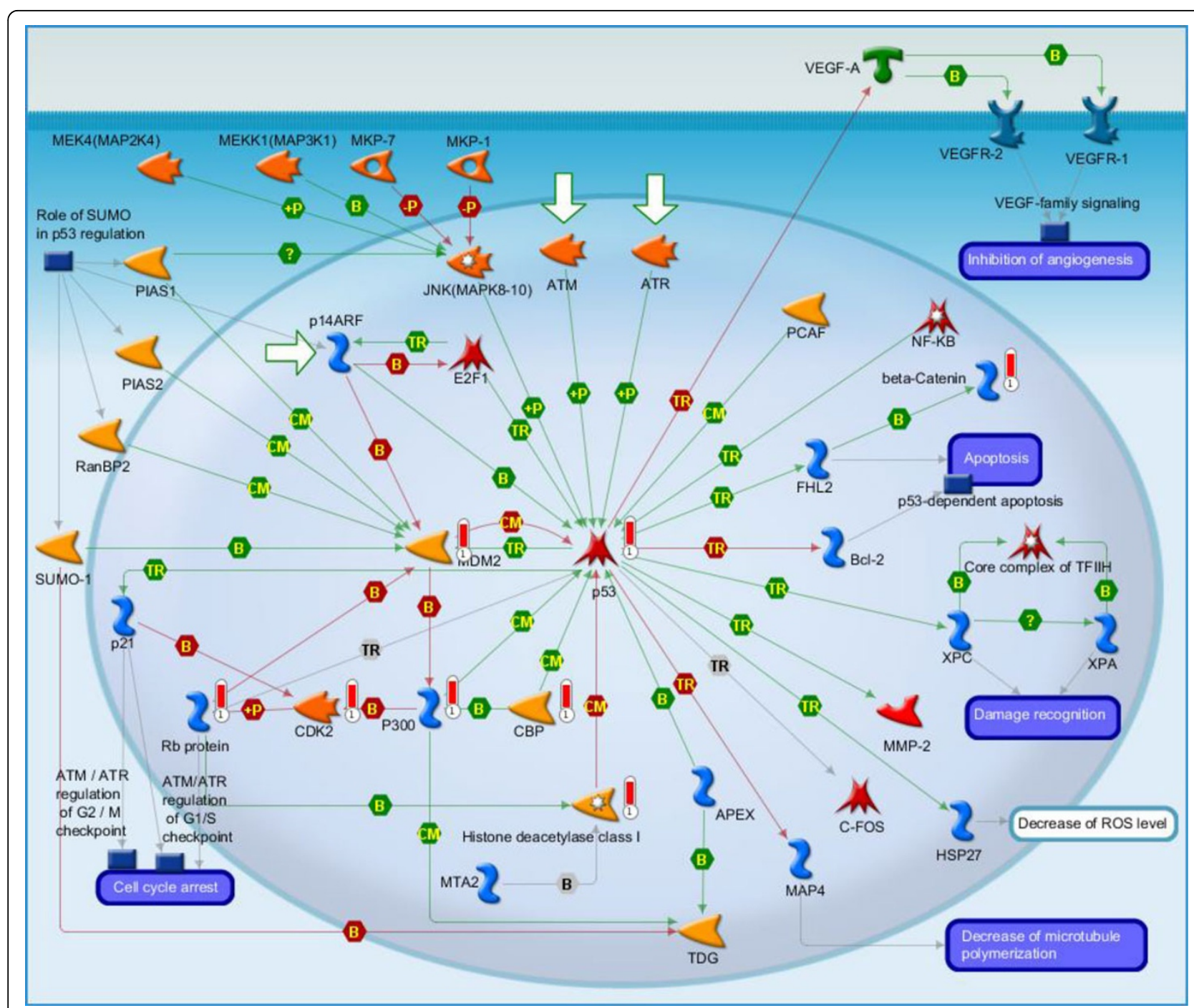

Figure 4 Transcription_P53 signaling pathway. It is the second highest scoring map (i.e., the map with the second lowest p-value). The Tumor protein p53, also known as p53 ortransformation-related protein 53 (TRP53), plays a significant role in shielding the genome integrity. While being activated, p53 will bind to the enhancer/promoter regions of downstream target genes. And then it regulates the transcription of these genes, through which it initiates cellular processes that responsible for lots of its tumor-suppressor related functions. It is not surprising that core network of 4 cancers are related to the p53 signaling pathway.

virtually screen the whole ZINC database using our IBM server with 160 cores. We have even performed virtual screening for Src inhibitors. However, constructing a proper HypoGen model is also very time-consuming. As an initial study using a novel approach, we used relatively unrestricted conditions to set up a rough HypoGen model. It would be beneficial to do virtual screening on another ligand database in the future.

Figure 7 Additional file 10 lists the total 54 ligands used to construct the HypoGen pharmacophore model. We present the structure of the pharmacophore in Figure 6 new, where NSC-625869 refers to the ligand in the NCI library of anti-cancer drugs that was screened and matched by this pharmacophore. The numerical results of the HypoGen model are shown in Tables 8, 9, 10. After having constructed this pharmacophore, we were able to use it to screen against other ligand databases, such as the Life Chemical database. Figure 7 and Table 11new show the compounds in the Life Chemical database screened and matched by this pharmacophore. Compared to the HypoGen model, the pharmacophore model derived by PharmGist is easier to obtain because PharmGist does not require the $\mathrm{IC}_{50}$ values of the compounds for computation. We have listed the results in Figure 8 and Table 12.

\section{Biological Experimental Validations}

Drug combination- Gefitinib (Iressa) combine with L4 and N4 in three different cells. In the three cell lines, 


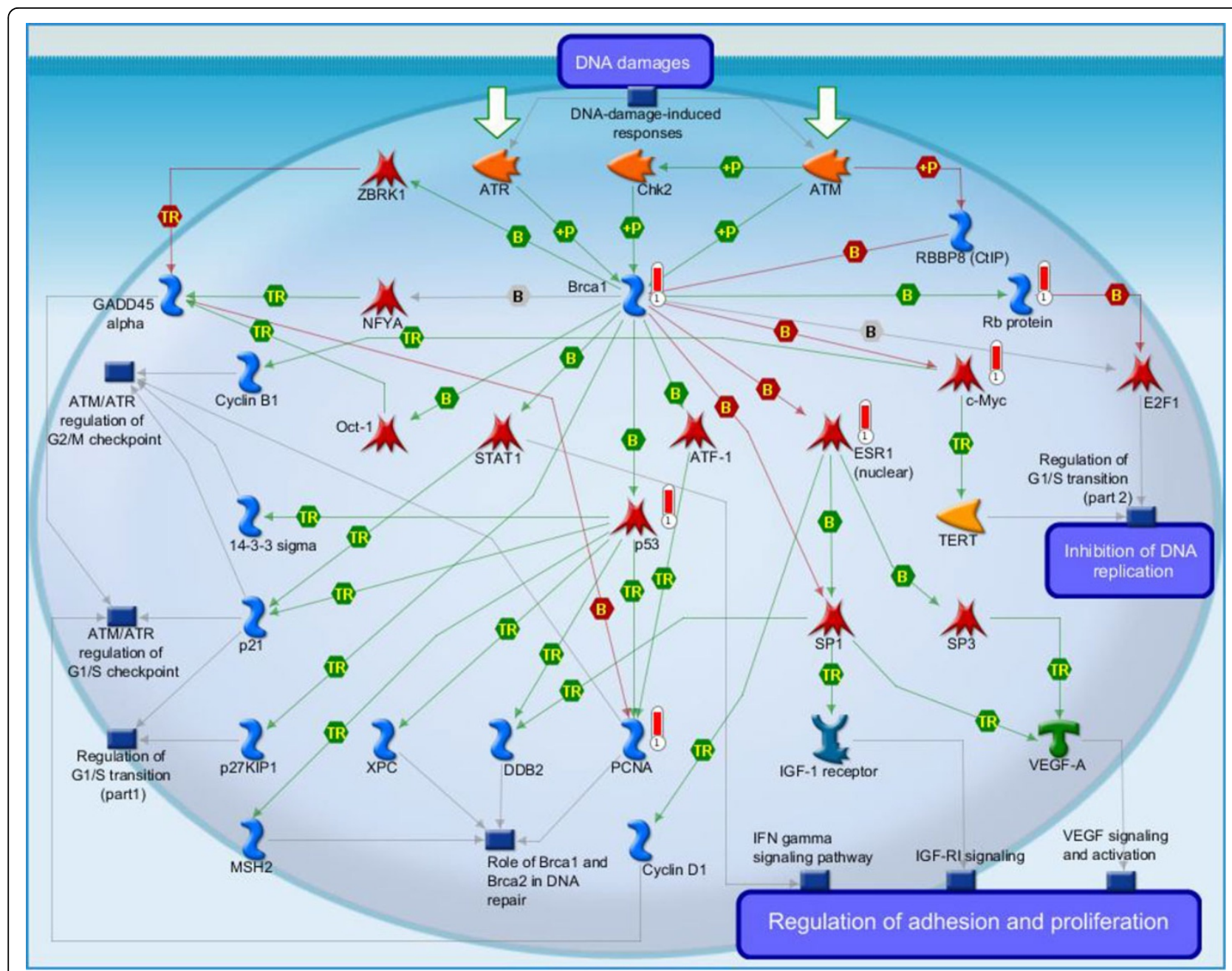

Figure 5 DNA damage_Brca1 as a transcription regulator. It is the third highest scoring map (i.e., the map with the third lowest p-value). Activation of breast cancer susceptibility gene 1 (BRCA1) by DNA damage occurs via activating the ataxia telangiectasia mutated serine/ threonine protein kinase (ATM) or serine/threonine-protein kinase ATR (ataxia telangiectasia and Rad3 related protein kinase). These protein kinases can either directly or indirectly phosphorylate BRCA1 (by cell cycle checkpoint kinase 2 (Chk2)). BRCA1 acts a significant role in the DNA repair process by expediting cellular response upon DNA repair. There are numerous DNA repair pathways (see map 427 Role of BRCA1 and BRCA2 in DNA repair). We know that DNA repair is highly related to various cancers.

they show that combination drug always get a better efficiency that only single Iressa. Please refer to Additional file 8 . At this stage, we have only used a simple pathway diagram to visualize the relationship between EGFR and Src without a large-scale systems biological analysis. These results gave us confidence that multiple drugs attacking multiple targets will achieve better results. Indeed, we have accumulated lots of similar results.

\section{Novel model combined with both systems biology and CADD}

- General demonstration: Our method has reversed the normal procedure of CADD with the help of systems biology. In the traditional CADD method, a single validated target, such as Src, EGFR, and FAK, is used as the protein target. The first step is usually virtual screening by docking or the pharmacophore method. The second step is to validate the top compounds from virtual screening with ELISA or Western blot wet-lab experiments. If they are found to bind to the target proteins, then researchers could employ these ligands in cell proliferation experiments. For our novel approach, we choose the top compounds from several targets simultaneously, and then perform the cell proliferations experiment at the beginning. Our hypothesis is that these drug cocktails would destroy the core network, although we have not experimentally validated whether each top compound binds to its corresponding target or not 

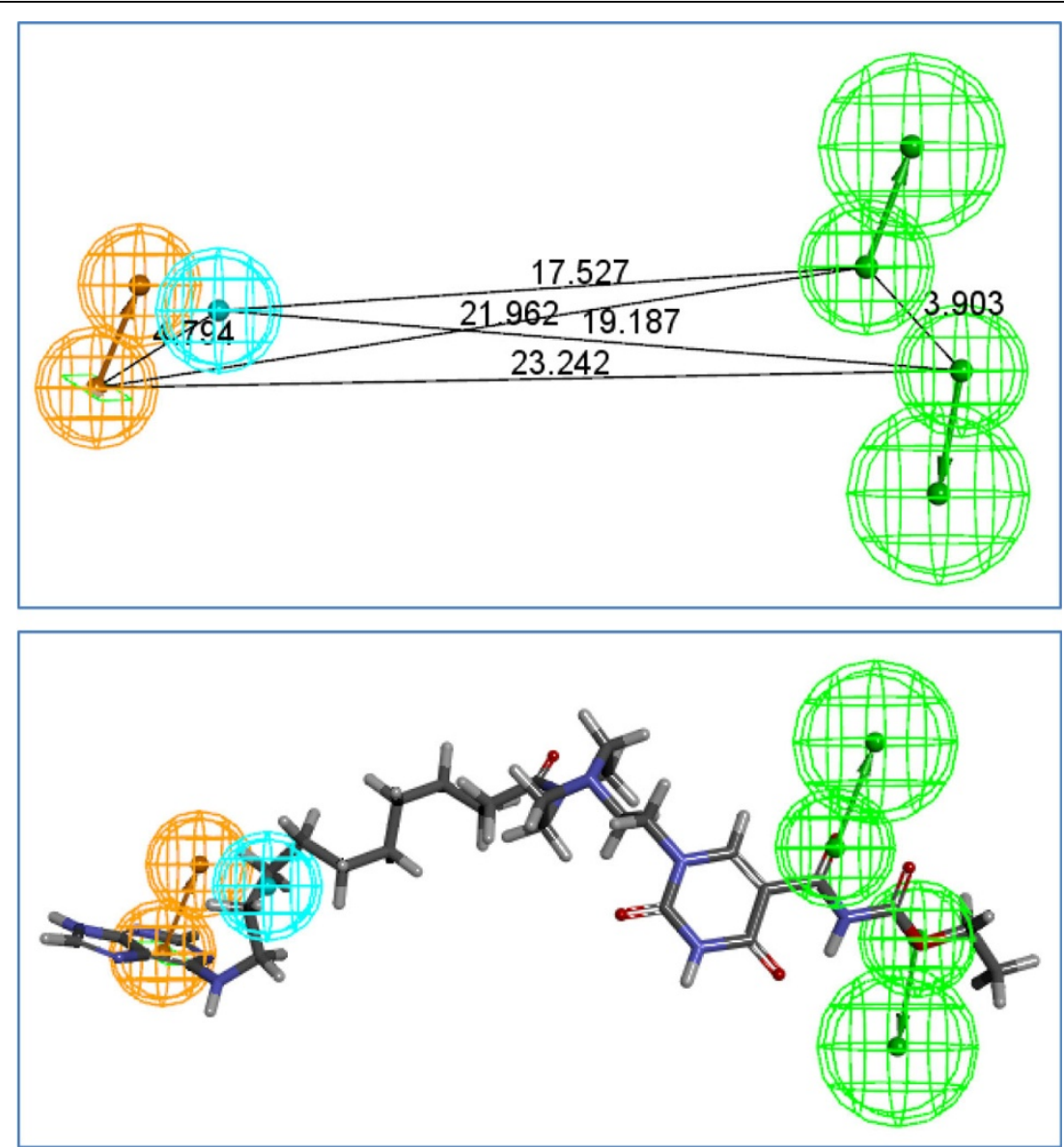

Figure 6 new 8- The hypogen pharmacophore model built by DS3.5. We show the distances between the pharmacophores. No. 625869 is the drug in $\mathrm{NCl}$ anti-cancer library screened and matched by this pharmacophore.

\begin{tabular}{|c|c|c|c|}
\hline Mapping & ID number & Fit Value & Estimate IC50 (nM) \\
\hline & F0325-0146 & 6.580 & 4.599 \\
\hline & F0289-0199 & 6.446 & 6.266 \\
\hline & F0922-0370 & 5.935 & 20.327 \\
\hline & F0012-0228 & 5.690 & 35.775 \\
\hline & F0496-0019 & 5.623 & 41.719 \\
\hline
\end{tabular}

Figure 7 new 9- Life-Chemical drugs screened out by Hypogen. These are the top ligands of Life-Chemical drug library screened by our hypogen pharmacophore model. Please see Table 10 for the detailed descriptions of these mapping results. 
Table 8. Top pharmacophore description

\begin{tabular}{lcccc}
\hline Category & Number of ligands & $\begin{array}{c}\mathbf{I C}_{50} \text { range } \\
(\mathbf{n M})\end{array}$ & Feature & Correlation \\
\hline 28 targets & 54 & $2-50000$ & HBA, HBA, HY, RA & 0.92 \\
\hline
\end{tabular}

Table 9. Top 10 pharmacophore descriptions

\begin{tabular}{|c|c|c|c|c|c|}
\hline Hypothesis & Total cost & $\Delta$ Cost & RMS & Correlation ( $r$ ) & Features \\
\hline 1 & 211.414 & 33.787 & 0.583549 & 0.924995 & $\mathrm{HBA}, \mathrm{HBA}, \mathrm{HY}, \mathrm{RA}$ \\
\hline 2 & 211.745 & 33.456 & 0.599912 & 0.920410 & HBA, HBA, HY, RA \\
\hline 3 & 212.295 & 32.906 & 0.606595 & 0.918758 & $\mathrm{HBA}, \mathrm{HBA}, \mathrm{HY}, \mathrm{RA}$ \\
\hline 4 & 212.530 & 32.671 & 0.609627 & 0.917975 & HBA, HBA, HY, RA \\
\hline 5 & 213.564 & 31.637 & 0.641692 & 0.908625 & HBA, HBA, HY, RA \\
\hline 6 & 213.870 & 31.331 & 0.651935 & 0.905497 & HBA, HBA, HY, RA \\
\hline 7 & 214.412 & 30.789 & 0.669021 & 0.900153 & $\mathrm{HBA}, \mathrm{HBA}, \mathrm{HY}, \mathrm{RA}$ \\
\hline 8 & 215.039 & 30.162 & 0.690558 & 0.893115 & HBA, HBA, HY, RA \\
\hline 9 & 215.282 & 29.919 & 0.694361 & 0.891929 & HBA, HBA, HY, RA \\
\hline 10 & 215.758 & 29.443 & 0.713440 & 0.885327 & HBA, HBA, HY, RA \\
\hline
\end{tabular}

$*$ Null cost $=245.201$; Fixed cost $=202.014$; Configuration cost $=19.2545 ; \Delta$ Cost $=$ Null cost - Total cost. All costs are in units of bits.

* HBA, hydrogen bond acceptor; HY, hydrophobic; RA, ring aromatic.

Table 10. Experimental and estimation values of $\mathrm{IC}_{50}$ for each of the 54 ligands

\begin{tabular}{|c|c|c|c|c|c|c|c|}
\hline $\mathrm{NCl}$ Drug & $\mathrm{IC}_{50}(\mathrm{nM})$ & Error & NCl Drug & $\mathrm{IC}_{50}(\mathrm{nM})$ & Error & & \\
\hline & Exp. & Est. & & & Exp. & Est. & \\
\hline 625869 & 2.0 & 2.4 & 1.2 & 669299 & 4792.2 & 5656.4 & 1.2 \\
\hline 668448 & 66.3 & 142.2 & 2.1 & 641847 & 5026.9 & 4480.2 & -1.1 \\
\hline 698233 & 256.5 & 97.5 & -2.6 & 669230 & 5282.5 & 4302.8 & -1.2 \\
\hline 668433 & 380.2 & 229.7 & -1.7 & 657381 & 5358.4 & 4451.3 & -1.2 \\
\hline 737026 & 810.9 & 366.4 & -2.2 & 643737 & 5376.8 & 4463.6 & -1.2 \\
\hline 654626 & 1377.0 & 1868.5 & 1.4 & 691369 & 5509.1 & 4822.7 & -1.1 \\
\hline 726771 & 1877.1 & 4272.0 & 2.3 & 627399 & 5527.5 & 4285.1 & -1.3 \\
\hline 717079 & 2067.7 & 4413.1 & 2.1 & 627727 & 5892.9 & 14154.0 & 2.4 \\
\hline 674086 & 2234.0 & 4138.9 & 1.9 & 693235 & 6107.2 & 5310.1 & -1.2 \\
\hline 666346 & 2270.0 & 5579.5 & 2.5 & 675823 & 6377.0 & 5049.0 & -1.3 \\
\hline 740601 & 2627.9 & 4280.5 & 1.6 & 665675 & 6591.5 & 4380.4 & -1.5 \\
\hline 639795 & 2742.6 & 4358.1 & 1.6 & 295632 & 7254.4 & 4402.1 & -1.6 \\
\hline 673172 & 2823.0 & 4721.0 & 1.7 & 668373 & 7390.1 & 4343.3 & -1.7 \\
\hline 641236 & 2827.4 & 4418.8 & 1.6 & 749 & 7534.5 & 21062.2 & 2.8 \\
\hline 76955 & 2836.7 & 4936.6 & 1.7 & 689447 & 8003.5 & 5397.9 & -1.5 \\
\hline 719481 & 3033.7 & 6903.6 & 2.3 & 202537 & 12700.6 & 21711.5 & 1.7 \\
\hline 406433 & 3064.1 & 12371.9 & 4.0 & 639906 & 13142.7 & 5880.0 & -2.2 \\
\hline 683481 & 3344.0 & 4540.1 & 1.4 & 622153 & 14753.4 & 5742.3 & -2.6 \\
\hline 682236 & 3395.0 & 4677.1 & 1.4 & 622154 & 15831.2 & 4771.3 & -3.3 \\
\hline 661908 & 3469.7 & 4310.7 & 1.2 & 692400 & 50000.0 & 13059.1 & -3.8 \\
\hline 633409 & 3661.4 & 4382.2 & 1.2 & 687325 & 50000.0 & 14121.3 & -3.5 \\
\hline 672968 & 3845.3 & 4363.1 & 1.1 & 689530 & 50000.0 & 17997.5 & -2.8 \\
\hline 680359 & 4140.3 & 4290.9 & 1.0 & 691200 & 50000.0 & 21974.7 & -2.3 \\
\hline 76519 & 4334.6 & 3133.5 & -1.4 & 684143 & 50000.0 & 32999.6 & -1.5 \\
\hline 702125 & 4732.2 & 4288.4 & -1.1 & 683630 & 50000.0 & 40400.2 & -1.2 \\
\hline 678636 & 4732.9 & 12631.9 & 2.7 & 694265 & 50000.0 & 51746.7 & 1.0 \\
\hline 682086 & 4770.1 & 4479.7 & -1.1 & 695571 & 50000.0 & 61122.2 & 1.2 \\
\hline
\end{tabular}

${ }^{*} \operatorname{Exp}=$ Experimental value; Est=Estimation value by pharmacophore; Error=Est./Exp. 
Table 11. The Life-Chemical ligands are virtually screened by the hypogen model

\begin{tabular}{|c|c|c|c|c|c|c|}
\hline ID No. & FitValue & Estimate & HBA_1 & HBA_2 & HY_3 & RA_4 \\
\hline F0325-0146 & 6.580 & 4.599 & 1 & 1 & 1 & 1 \\
\hline F0289-0199 & 6.446 & 6.266 & 1 & 1 & 1 & 1 \\
\hline F0922-0370 & 5.935 & 20.327 & 1 & 1 & 1 & 1 \\
\hline F0012-0228 & 5.690 & 35.775 & 1 & 1 & 1 & 1 \\
\hline F0496-0019 & 5.623 & 41.719 & 1 & 1 & 1 & 1 \\
\hline F0737-0405 & 5.55006 & 49.3217 & 1 & 1 & 1 & 1 \\
\hline F0382-0020 & 5.53538 & 51.0174 & 1 & 1 & 1 & 1 \\
\hline F0325-0148 & 5.1207 & 132.555 & 1 & 1 & 1 & 1 \\
\hline F0737-0312 & 5.00395 & 173.442 & 1 & 1 & 1 & 1 \\
\hline F0725-0356 & 4.98991 & 179.137 & 1 & 1 & 1 & 1 \\
\hline F0301-0263 & 4.9208 & 210.04 & 1 & 1 & 1 & 1 \\
\hline F0473-0314 & 4.90716 & 216.74 & 1 & 1 & 1 & 1 \\
\hline F0922-0913 & 4.87165 & 235.207 & 1 & 1 & 1 & 1 \\
\hline F1601-0068 & 4.58408 & 456.055 & 1 & 1 & 1 & 1 \\
\hline F0463-0195 & 4.50862 & 542.605 & 1 & 1 & 1 & 1 \\
\hline F0866-0317 & 4.26226 & 956.832 & 1 & 1 & 1 & 1 \\
\hline F0537-0936 & 4.17732 & $1,163.54$ & 1 & 1 & 1 & 1 \\
\hline F0180-0144 & 3.96884 & $1,880.45$ & 1 & 1 & 1 & 1 \\
\hline F0922-0900 & 3.95736 & 1,930.82 & 1 & 1 & 1 & 1 \\
\hline
\end{tabular}

Please see the mapping diagrams in Figure 11.

- Time complexity versus space complexity: Our novel approach is some kind like the problem of algorithm about the time complexity and space complexity. To attack each protein one by one is similar to the problem of time complexity. This is the traditional strategy for single target drug design. The traditional method needs to make sure the drugs target to which protein exactly. In the language of computer science, the traditional CADD method can only do serial work but not parallelization work. By the help of our systems biology approach, it turns the origin problem from time complexity to space complexity. That is, you can do parallelization attack to the multiple proteins simultaneously. The powerful of this parallelization attack is that you can do various drugs combination trial at the short time. When you get the better results, you can go back to check each drug in the combination is useful for the proteins individually or not. Honest to speak, there are possible too many uncertainties and inaccuracies in this model, such as the network biomarkers predicted by our systems biology approach, the binding site information, the pharmacophore model or even the predicted 3D protein structures. However, due to the powerful of parallelization attack (cocktail multiple drugs), it is possible to find the useful cocktail combination under the situation of so many uncertainties and inaccuracies. The problem of space complexity we encounter now is that how many drugs we could use at the same time. And this is our feature work.

-Welcome to asking for our help and cooperation: We must emphasize that no matter how powerful of our model, the most important thing is to combine with the correct medical knowledge and intuition. After our model gives so many possible combinations of drug, you have to decide a set of best ones with your medical knowledge and intuition to do the wet-lab experiment validation. We already have a lot of these wet-lab results but not published yet. Our teams also have abundant experience on the single target drug design [45-47]. We have also built a webserver of the systems biology model in the research but not opened to public yet. It is welcome for your feedback and cooperation. You are welcome to modify the old version source code of our systems biology model [48]. Please refer to Additional file 8.

- Novelty and expectation results in the future: Combination drugs is not a novel idea [49,50]. To design combination drugs by our systems biology and CADD methods is a novel work. We expect many combination drugs will be really useful by the help of model. We would like to enhance and modify our model in the future.

\section{Availability of this method}

We are in the process of building a webserver to identify network biomarkers. At this stage, we can construct the network on request. We also offer a working version of the source code (Additional File 11), and the readers can modify this version of source code to perform their research experiments.

\section{Conclusions}

In this study, we combined systems biology with traditional computational-aided drug design to design drug 


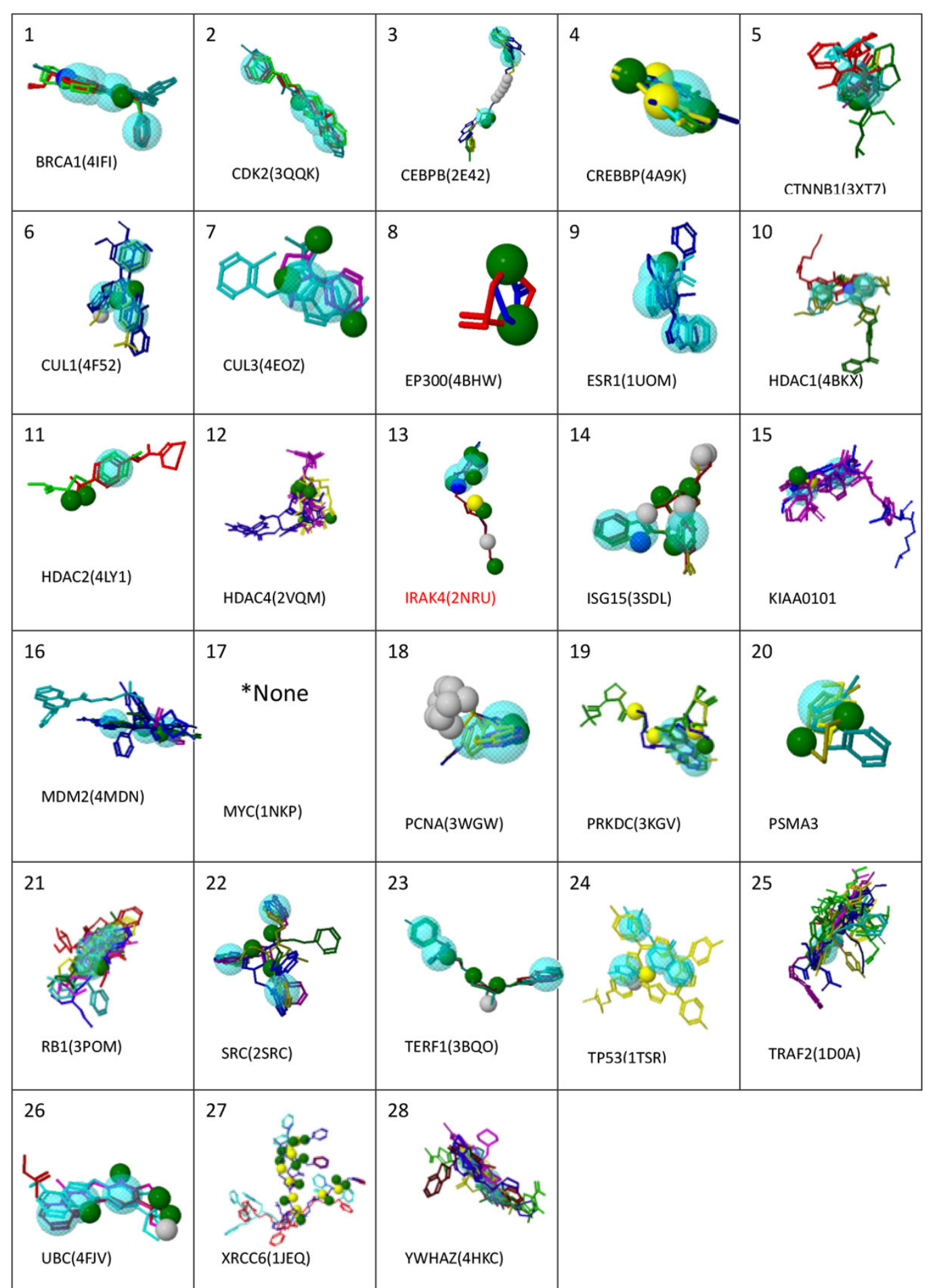

Figure 8 new 10-PharmGist Pharmacophore Model. The pharmacophores for each of the 28 significant proteins built by PharmGist. Detailed descriptions of these pharmacophores are in Tables 7-9. [*17:MYC(1NKP)'s pharmacophore could not be generated.]

cocktails to inhibit the 28 proteins that comprise the common core network markers of four cancers. The results of our previous study have led us to believe that those proteins likely represent house-keeping proteins of these four cancers. Wet-lab researchers could use the cocktails of multiple drugs indicated by our analysis as objects of study in experiments on treating the four cancers. Moreover, with the help of sensitivity analysis, we 
Table 12. The pharmacophores descriptions of PharmGist

\begin{tabular}{|c|c|c|c|c|c|c|c|c|c|c|c|c|c|}
\hline No. & Protein & PDB & Score & Features & Spatial Features & $\mathbf{R}$ & $\mathrm{H}$ & $\mathrm{D}$ & A & $\mathrm{N}$ & $P$ & Chemicals & Fig \\
\hline 1 & BRCA1 & $4 \mid \mathrm{FI}$ & 39.2 & 7 & 7 & 4 & 0 & 0 & 2 & 0 & 1 & 3 & $9-1$ \\
\hline 2 & CDK2 & 3QQK & 33.1 & 5 & 5 & 4 & 0 & 0 & 1 & 0 & 0 & 3 & $9-2$ \\
\hline 3 & CEBPB & $2 \mathrm{E} 42$ & 40.4 & 12 & 12 & 3 & 5 & 0 & 4 & 0 & 0 & 3 & $9-3$ \\
\hline 4 & CREBBP & $4 \mathrm{~A} 9 \mathrm{~K}$ & 29.9 & 5 & 5 & 1 & 0 & 2 & 2 & 0 & 0 & 9 & $9-4$ \\
\hline 5 & CTNNB1 & $3 \mathrm{TX7}$ & 23.7 & 3 & 3 & 2 & 0 & 0 & 1 & 0 & 0 & 7 & $9-5$ \\
\hline 6 & CUL1 & $4 \mathrm{~F} 52$ & 37.5 & 7 & 7 & 4 & 1 & 0 & 2 & 0 & 0 & 3 & $9-6$ \\
\hline 7 & CUL3 & 4EOZ & 25.7 & 5 & 5 & 2 & 0 & 0 & 3 & 0 & 0 & 3 & $9-7$ \\
\hline 8 & EP300 & $4 \mathrm{BHW}$ & 3.0 & 2 & 2 & 0 & 0 & 0 & 2 & 0 & 0 & 2 & $9-8$ \\
\hline 9 & ESR1 & $1 \cup O M$ & 33.1 & 5 & 5 & 4 & 0 & 0 & 1 & 0 & 0 & 3 & $9-9$ \\
\hline 10 & HDAC1 & $4 B K X$ & 26.5 & 4 & 4 & 3 & 0 & 0 & 0 & 0 & 1 & 4 & $9-10$ \\
\hline 11 & HDAC2 & $4 \mathrm{LY} 1$ & 6.0 & 3 & 3 & 1 & 0 & 0 & 2 & 0 & 0 & 2 & $9-11$ \\
\hline 12 & HDAC4 & 2VQM & 34.0 & 7 & 5 & 0 & 0 & 2 & 5 & 0 & 0 & 3 & $9-12$ \\
\hline 13 & IRAK4 & 2NRU & 44.5 & 13 & 11 & 2 & 1 & 3 & 6 & 0 & 1 & 3 & $9-13$ \\
\hline 14 & ISG15 & 3SDL & 42.7 & 16 & 14 & 3 & 7 & 2 & 3 & 0 & 1 & 3 & $9-14$ \\
\hline 15 & KIAA0101 & & 26.5 & 4 & 4 & 2 & 0 & 1 & 1 & 0 & 0 & 3 & $9-15$ \\
\hline 16 & MDM2 & $4 \mathrm{MDN}$ & 36.0 & 5 & 5 & 3 & 0 & 0 & 2 & 0 & 0 & 6 & $9-16$ \\
\hline 17 & MYC & 1NKP & & & & & & & & & & & $9-17$ \\
\hline 18 & PCNA & $3 W G W$ & 36.0 & 14 & 14 & 3 & 9 & 0 & 2 & 0 & 0 & 3 & $9-18$ \\
\hline 19 & PRKDC & 3KGV & 36.7 & 7 & 7 & 3 & 0 & 3 & 1 & 0 & 0 & 3 & $9-19$ \\
\hline 20 & PSMA3 & & 14.7 & 3 & 3 & 1 & 0 & 0 & 2 & 0 & 0 & 3 & $9-20$ \\
\hline 21 & RB1 & ЗРОМ & 36.4 & 4 & 4 & 3 & 0 & 0 & 1 & 0 & 0 & 9 & $9-21$ \\
\hline 22 & SRC & $2 S R C$ & 35.7 & 6 & 6 & 3 & 0 & 0 & 3 & 0 & 0 & 4 & $9-22$ \\
\hline 23 & TERF1 & 3BQO & 36.5 & 7 & 7 & 3 & 1 & 0 & 3 & 0 & 0 & 4 & $9-23$ \\
\hline 24 & TP53 & $1 \mathrm{TSR}$ & 33.8 & 6 & 6 & 4 & 1 & 1 & 0 & 0 & 0 & 3 & $9-24$ \\
\hline 25 & TRAF2 & 1D0A & 30.9 & 3 & 3 & 2 & 0 & 0 & 1 & 0 & 0 & 16 & $9-25$ \\
\hline 26 & UBC & 4FJV & 41.6 & 10 & 9 & 3 & 1 & 1 & 5 & 0 & 0 & 3 & $9-26$ \\
\hline 27 & XRCC6 & 1JEQ & 141.1 & 22 & 22 & 0 & 0 & 10 & 12 & 0 & 0 & 3 & $9-27$ \\
\hline 28 & YWHAZ & $4 H K C$ & 34.9 & 3 & 3 & 3 & 0 & 0 & 0 & 0 & 0 & 12 & $9-28$ \\
\hline
\end{tabular}

Their pharmacophore diagrams are shown in Figure 7.

also found the most likely multiple drug targets for each individual cancer.

\section{Additional material}

Additional file 1: Parameter Identification of PPI network by Maximum Likelihood Method.

Additional file 2: Determination of significant protein associations by AIC and Student's t-test.

Additional file 3: The identified significant proteins in 4 cancers

Additional file 4: Docking results for the top 20 ligands for the 28 proteins studied

Additional file 5: new 6: User interface of commercial software and free webserver

Additional file 6: new 7: Mathematical foundation of Metacore.

Additional file 7: new 5: Binding site information for the 28 proteins.

Additional file 8: new 10: Biological experimental validation.

Additional file 9: new $\mathbf{8}$. Docking pose analysis of $\mathbf{2 8}$ core proteins. The docking poses analysis shows the key residues of the proteins which interact with the ligands. The analysis reveals more mechanisms to help us design de novo drugs.
Additional file 10: new 9. 54 ligands used for developing the Hypogen model.

Additional file 11: Source code ( ${ }^{*}$.rar).

Competing interests

The authors declare that they have no competing interests.

\section{Authors' contributions}

BSC and YHW designed and conduct the theory and experiments of systems biology. CWL modified the core network. JC, YHW, CLL and TSC design and perform the CADD. JC, CAC, PSJ and YHL design and perform the wet-lab biological experiments. LJC perform the pathway and functional analysis. BSC and YHW integrated the whole work. YHW drafted the manuscript. All of the authors have approved the publication of the manuscript.

\section{Acknowledgements}

The authors are grateful for the support provided by the Ministry of Science and Technology, Taiwan through grants MOST-103-2745-E-007-001-ASP, MOST-103-2221-E-038 -013 -MY2, and MOST-104-2218-E-007-021.

\section{Declaration}

Funding for this publication was provided by the Ministry of Science and Technology, Taiwan through grants MOST-103-2745-E-007-001-ASP, MOST103-2221-E-038 -013 -MY2, and MOST-104-2218-E-007-021. 
This article has been published as part of BMC Medical Genomics Volume 8 Supplement 4, 2015: Joint 26th Genome Informatics Workshop and 14th International Conference on Bioinformatics: Medical genomics. The full contents of the supplement are available online at http://www. biomedcentral.com/bmcmedgenomics/supplements/8/S4.

\section{Authors' details}

'Laboratory of Control and Systems Biology, Department of Electrical Engineering, National Tsing Hua University, Hsinchu 30013, Taiwan. ${ }^{2}$ Biomedical Technology and Device Research Laboratories, Industrial Technology Research Institute, Hsinchu, Taiwan, ROC. ${ }^{3}$ Institute of Biomedical Science, National Chung Hsing University, Taiwan 40227, Republic of China. ${ }^{4}$ Molecular Medicine Research Center, Chang Gung University, Taoyuan, Taiwan, ROC.

Published: 9 December 2015

\section{References}

1. Sager R: Expression genetics in cancer: shifting the focus from DNA to RNA. Proceedings of the National Academy of Sciences 1997, 94(3):952-955.

2. Hanahan D, Weinberg RA: The hallmarks of cancer. Cell 2000, 100(1):57-70.

3. Hanahan D, Weinberg RA: Hallmarks of cancer: the next generation. Cell 2011, 144(5):646-674

4. Nibbe RK, Koyutürk M, Chance MR: An integrative-omics approach to identify functional sub-networks in human colorectal cancer. PLoS computational biology 2010, 6(1):e1000639.

5. Zhang X, Wei D, Yap Y, Li L, Guo S, Chen F: Mass spectrometry-based "omics" technologies in cancer diagnostics. Mass spectrometry reviews 2007, 26(3):403-431.

6. Chen $B S, L i C W$ : Analysing microarray data in drug discovery using systems biology. Expert Opin Drug Discov 2007, 2(5):755-768.

7. Randhawa V, Bagler G: Identification of SRC as a potent drug target for asthma, using an integrative approach of protein interactome analysis and in silico drug discovery. Omics: a journal of integrative biology 2012, 16(10):513-526.

8. Gupta A, Bhunia S, Balaramnavar V, Saxena A: Pharmacophore modelling, molecular docking and virtual screening for EGFR (HER 1) tyrosine kinase inhibitors. SAR and QSAR in Environmental Research 2011, 22(34):239-263.

9. Huang H-J, Lee K-J, Yu HW, Chen C-Y, Hsu C-H, Chen H-Y, Tsai F-J, Chen CY-C: Structure-based and ligand-based drug design for HER 2 receptor. Journal of Biomolecular Structure and Dynamics 2010, 28(1):23-37.

10. Pirhadi S, Ghasemi JB: 3D-QSAR analysis of human immunodeficiency virus entry-1 inhibitors by CoMFA and CoMSIA. European journal of medicinal chemistry 2010, 45(11):4897-4903.

11. Fernandez M, Caballero J, Fernandez L, Sarai A: Genetic algorithm optimization in drug design QSAR: Bayesian-regularized genetic neural networks (BRGNN) and genetic algorithm-optimized support vectors machines (GA-SVM). Molecular diversity 2011, 15(1):269-289.

12. Kinnings SL, Liu N, Tonge PJ, Jackson RM, Xie L, Bourne PE: A machine learning-based method to improve docking scoring functions and its application to drug repurposing. Journal of chemical information and modeling 2011, 51(2):408-419.

13. Zhao X, Li Q, Bian L, Zheng X, Zheng J, Zhang Y, Li Z: Using immobilized G-protein coupled receptors to screen bioactive traditional Chinese medicine compounds with multiple targets. Journal of pharmaceutical and biomedical analysis 2012, 70:549-552.

14. Chen B-S, Hsu C-Y, Liou J-J: Robust design of biological circuits: evolutionary systems biology approach. BioMed Research International 2011.

15. Tu C-T, Chen B-S: New measurement methods of network robustness and response ability via microarray data. PLoS One 2013, 8(1):e55230.

16. Ivanov AA, Khuri FR, Fu HA: Targeting protein-protein interactions as an anticancer strategy. Trends Pharmacol Sci 2013, 34(7):393-400.

17. Wong YH, Chen RH, Chen BS: Core and specific network markers of carcinogenesis from multiple cancer samples. J Theor Biol 2014, 362:17-34.

18. Lamptey P, Cates W Jr: Opinion: An Ounce of Prevention is Worth a Million Lives. AIDSCaptions 1997.

19. White AW, Westwell AD, Brahemi G: Protein-protein interactions as targets for small-molecule therapeutics in cancer. Expert Rev Mol Med 2008, 10:e8.

20. Johansson R: System Modeling and Identification 1993.
21. Pagano M, Gauvreau K: Principles of biostatistics 2000.

22. Wang YC, Chen BS: A network-based biomarker approach for molecular investigation and diagnosis of lung cancer. BMC Med Genomics 2011, 4:2.

23. Ashburner M, Ball CA, Blake JA, Botstein D, Butler H, Cherry JM, Davis AP, Dolinski K, Dwight SS, Eppig JT, et al: Gene ontology: tool for the unification of biology. The Gene Ontology Consortium Nat Genet 2000, 25(1):25-29.

24. Huang da W, Sherman BT, Lempicki RA: Systematic and integrative analysis of large gene lists using DAVID bioinformatics resources. Nat Protoc 2009, 4(1):44-57.

25. Huang DW, Sherman BT, Tan Q, Kir J, Liu D, Bryant D, Guo Y, Stephens R, Baseler MW, Lane HC, et al: DAVID Bioinformatics Resources: expanded annotation database and novel algorithms to better extract biology from large gene lists. Nucleic Acids Res 2007, 35(Web Server):W169-175.

26. Kanehisa M, Goto S, Sato Y, Kawashima M, Furumichi M, Tanabe M: Data, information, knowledge and principle: back to metabolism in KEGG. Nucleic Acids Res 2014, 42(Database):D199-205.

27. Kanehisa M, Goto S: KEGG: kyoto encyclopedia of genes and genomes. Nucleic Acids Res 2000, 28(1):27-30.

28. Mi H, Muruganujan A, Thomas PD: PANTHER in 2013: modeling the evolution of gene function, and other gene attributes, in the context of phylogenetic trees. Nucleic Acids Research 2013, 41(Database):D377-386

29. MetaCore MetaCore Bioinformatics software from Thomson Reuters. [https://portal.genego.com/].

30. MetaCore Advanced Training Manual. [https://portal.genego.com/help/ MetaCore_Advanced_Training_Manual_5.0.pdf].

31. MetaCore Training Manual. [https://portal.genego.com/help/ MetaCore_bio_manual_5.0.pdf].

32. Life Chemicals Inc. sells its products and services to more than 2000 customers, with its sales volume steadily growing. [http://www. lifechemicals.com/.

33. Zhang Y: I-TASSER server for protein 3D structure prediction. BMC Bioinformatics 2008, 9:40.

34. Yang JY, Roy A, Zhang Y: Protein-ligand binding site recognition using complementary binding-specific substructure comparison and sequence profile alignment. Bioinformatics 2013, 29(20):2588-2595.

35. Schneidman-Duhovny D, Dror O, Inbar Y, Nussinov R, Wolfson HJ: PharmaGist: a webserver for ligand-based pharmacophore detection. Nucleic Acids Research 2008, 36(Web Server):W223-228.

36. Yi-Hua Lai M-HC, Sih-Yin Lin, Sheng-Yi Lin, Yung-Hao Wong, Sung-Liang Yu, Huei-Wen Chen, Chih-Hsin Yang, Gee-Chen Chang, Jeremy JWChen: Rhodomycin A, a novel Src-targeted compound, can suppress lung cancer cell progression via modulating Src-related pathways. Oncotarget 2015.

37. Sancar A, Lindsey-Boltz LA, Unsal-Kacmaz K, Linn S: Molecular mechanisms of mammalian DNA repair and the DNA damage checkpoints. Annual review of biochemistry 2004, 73:39-85.

38. Bakkenist CJ, Kastan MB: DNA damage activates ATM through intermolecular autophosphorylation and dimer dissociation. Nature 2003, 421(6922):499-506

39. Jin S, Levine AJ: The p53 functional circuit. Journal of cell science 2001, 114(Pt 23):4139-4140.

40. Cortez D, Wang Y, Qin J, Elledge SJ: Requirement of ATM-dependent phosphorylation of BRCA1 in the DNA damage response to doublestrand breaks. Science 1999, 286(5442):1162-1166.

41. Tibbetts RS, Cortez D, Brumbaugh KM, Scully R, Livingston D, Elledge SJ, Abraham RT: Functional interactions between BRCA1 and the checkpoint kinase ATR during genotoxic stress. Genes \& development 2000, 14(23):2989-3002.

42. Zhang J, Willers H, Feng Z, Ghosh JC, Kim S, Weaver DT, Chung JH, Powell SN, Xia F: Chk2 phosphorylation of BRCA1 regulates DNA doublestrand break repair. Molecular and cellular biology 2004, 24(2):708-718.

43. Fleck O, Nielsen O: DNA repair. Journal of cell science 2004, 117(Pt 4):515-517.

44. Irwin JJ, Sterling T, Mysinger MM, Bolstad ES, Coleman RG: ZINC: a free tool to discover chemistry for biology. J Chem Inf Model 2012, 52(7):1757-1768.

45. Yi-Hua Lai M-HC, Sih-Yin Lin, Sheng-Yi Lin, Yung-Hao Wong, Sung-Liang Yu, Huei-Wen Chen, Chih-Hsin Yang, Gee-Chen Chang, Jeremy J.W Chen: Rhodomycin A, a novel Src-targeted compound, can suppress lung cancer cell progression via modulating Src-related pathways. Oncotarget 2015. 
46. Hsueh YS, Lin CL, Chiang NJ, Yen CC, Li CF, Shan YS, Ko CH, Shih NY, Wang LM, Chen TS, et al: Selecting tyrosine kinase inhibitors for gastrointestinal stromal tumor with secondary KIT activation-loop domain mutations. PloS one 2013, 8(6):e65762.

47. Shih KC, Lin CY, Chi HC, Hwang CS, Chen TS, Tang CY, Hsiao NW: Design of novel FLT-3 inhibitors based on dual-layer 3D-QSAR model and fragment-based compounds in silico. Journal of chemical information and modeling 2012, 52(1):146-155.

48. Chu L-H, Chen B-S: Construction of a cancer-perturbed protein-protein interaction network for discovery of apoptosis drug targets. BMC systems biology 2008, 2(1):56

49. Maione P, Gridelli C, Troiani T, Ciardiello F: Combining targeted therapies and drugs with multiple targets in the treatment of NSCLC. The Oncologist 2006, 11(3):274-284.

50. Giordano S, Petrelli A: From single-to multi-target drugs in cancer therapy: when aspecificity becomes an advantage. Current medicinal chemistry 2008, 15(5):422-432.

doi:10.1186/1755-8794-8-S4-S4

Cite this article as: Wong et al:: Multiple target drug cocktail design for attacking the core network markers of four cancers using ligand-based and structure-based virtual screening methods. BMC Medical Genomics 2015 8(Suppl 4):S4.

\section{Submit your next manuscript to BioMed Central and take full advantage of:}

- Convenient online submission

- Thorough peer review

- No space constraints or color figure charges

- Immediate publication on acceptance

- Inclusion in PubMed, CAS, Scopus and Google Scholar

- Research which is freely available for redistribution

Submit your manuscript at www.biomedcentral.com/submit 